2018-04

\title{
Approximate Solutions for Ideal Dam-Break Sediment-Laden Flows on Uniform Slopes
}

\author{
$\mathrm{Ni}$, Yufang
}

http://hdl.handle.net/10026.1/17698

\subsection{2/2017wr021340}

Water Resources Research

American Geophysical Union (AGU)

All content in PEARL is protected by copyright law. Author manuscripts are made available in accordance with publisher policies. Please cite only the published version using the details provided on the item record or document. In the absence of an open licence (e.g. Creative Commons), permissions for further reuse of content should be sought from the publisher or author. 
1

3

\section{Approximate Solutions for Ideal Dam-Break Sediment-Laden Flows on Uniform Slopes}

\section{Yufang $\mathrm{Ni}^{1}$, Zhixian $\mathrm{Cao}^{1 \dagger}$, Alistair Borthwick ${ }^{2}$, and Qingquan $\mathrm{Liu}^{3}$}

${ }^{1}$ State Key Laboratory of Water Resources and Hydropower Engineering Science, Wuhan University, Wuhan, China.

${ }^{2}$ School of Engineering, The University of Edinburgh, Edinburgh EH9 3JL, UK.

${ }^{3}$ Department of Mechanics, Beijing Institute of Technology, Beijing, China.

${ }^{\dagger}$ Corresponding author: Zhixian Cao (zxcao@whu.edu.cn)

\section{Key Points:}

- Approximate solutions are derived for idealized dam-break sediment-laden flows on uniform slopes with negligible bed evolution

- The approximate solutions are shown to agree well with numerical solutions based on finite volume method

- This work facilitates benchmark solutions for verifying shallow water hydro-sedimentmorphodynamic models 


\section{Abstract}

Shallow water hydro-sediment-morphodynamic (SHSM) models have been applied increasingly widely in hydraulic engineering and geomorphological studies over the past few decades. Analytical and approximate solutions are usually sought to verify such models and therefore confirm their credibility. Dam-break flows are often evoked because such flows normally feature shock waves and contact discontinuities that warrant refined numerical schemes to solve. While analytical and approximate solutions to clear-water dam-break flows have been available for some time, such solutions are rare for sediment transport in dam-break flows. Here we aim to derive approximate solutions for ideal dam-break sediment-laden flows resulting from the sudden release of a finite volume of frictionless, incompressible water-sediment mixture on a uniform slope. The approximate solutions are presented for three typical sediment transport scenarios, i.e., pure advection; pure sedimentation; and concurrent entrainment and deposition. Although the cases considered in this paper are not real, the approximate solutions derived facilitate suitable benchmark tests for evaluating SHSM models, especially presently when shock waves can be numerically resolved accurately with a suite of finite volume methods, whilst the accuracy of the numerical solutions of contact discontinuities in sediment transport remains generally poorer.

\section{Introduction}

Over the past few decades, there have been widespread applications of shallow water hydro-sediment-morphodynamic (SHSM) models in hydraulic engineering and geomorphological studies (Cao et al., 2017). A wide range of fluvial processes have been investigated, including dam-break floods over erodible beds (Cao et al., 2004; Capart \& Young, 1998; Huang et al., 2012, 2014; Wu \& Wang, 2007; Xia et al., 2010) and morphodynamic processes (Nicholas et al., 2013; Qian et al., 2017; Wang et al., 2008; Wu, 2007; Xie, 1990). In fact, as pointed out by Cao et al. (2017), the SHSM equations have been extended to model coastal processes (Kim, 2015; Xiao et al., 2010; Zhu \& Dodd, 2015), watershed erosion processes (Kim et al., 2013), subaqueous sediment-laden flows and turbidity currents (Hu \& Cao, 2009; Hu et al., 2012), and sharply stratified processes (Li et al., 2013; Spinewine \& Capart, 2013; Zech et al., 2015). Most recently, shallow water two-phase models have been developed to better resolve fluvial processes (Cristo et al., 2015; Li, Cao, Qian, et al., 2017) and debris flows (Li, Cao, Hu, et al., 2017a, b). Pivotal to the success of SHSM models are the recent advances in both computer technology and numerical methods. Examples include developments in the finite difference method (Wu, 2007; Xie, 1990), the finite element method (Michoski et al., 2013), and the finite volume method (FVM) (Tan, 1998; Toro, 2001). Prior to applications, such mathematical models have to be either verified against analytical and approximate solutions or validated against experimental datasets for SHSM processes or pure hydrodynamic processes without involving sediment transport. However, laboratory experiments are time-consuming and measurement accuracy is often limited. In contrast, analytical and approximate solutions can be derived for simplified physical problems, of which the governing equations are mathematically 
tractable by analytical techniques (Fraccarollo \& Capart, 2002; Toro, 2001). Although it is inevitable to neglect some physical mechanisms to obviate mathematical difficulty, analytical and approximate solutions are particularly valuable for the systematic assessment of numerical models describing more complex and realistic problems (Ancey et al., 2008; Berthon et al., 2012; Fernandez-Feria, 2006; Hogg, 2006; Pritchard \& Hogg, 2002; Ritter, 1892; Stoker, 1957; Toro, 2001; Zoppou \& Roberts, 2003).

Dam-break flows are notable for their destructive power, which can cause catastrophic loss of life and substantial societal, economic and infrastructural damage (Cao et al., 2004). Dam-break flows are also of great interest to researchers investigating the ability of mathematical models to capture shock waves and contact discontinuities (Pritchard \& Hogg, 2002; Toro, 2001). Ancey et al. (2008) have summarized progress towards both analytical and approximate solutions for clear-water dam-break flows. In early stages, a variety of dam-break problems have been designed, including floods propagating over a frictionless horizontal bed (Hogg, 2006; Ritter, 1892; Stoker, 1957) and a rough horizontal bed (Dressler, 1952; Hogg \& Pritchard, 2004; Whitham, 1955). However, only asymptotic solutions could be developed for floods over a rough horizontal bed. Afterwards, cases with frictionless and inclined beds have been considered. Mangeney et al. (2000) studied debris avalanches and examined the case of a dam perpendicular to the bed holding back water of constant depth in a reservoir of infinite length. Ancey et al. (2008) and Antuono and Hogg (2009) considered a flood of finite volume; the dam was again perpendicular to the bed, rather than vertical, in order to prevent singular behavior occurring when the method of characteristics was employed. Fernandez-Feria (2006) attempted to derive analytical solutions for dam-break flow with a vertical dam on a uniform slope, yet the solutions were actually approximate as numerical realization was required..

To date, however, analytical and approximate solutions for sediment-laden dam-break flows are rare compared to their clear-water counterparts. Fraccarollo and Capart (2002) presented an approximate theoretical description of the formative stages of erosional dam-break flows over a horizontal bed, under the assumptions of equilibrium sediment transport and negligible bed resistance. Pritchard and Hogg (2002) derived exact solutions for suspended sediment transport under dam-break flows over a frictionless horizontal bed with an infinite volume of water upstream, assuming that the bed geometry was not altered. Berthon et al. (2012) provided an analytical solution of the shallow water system coupled to the Exner equation, which was used to update the bed elevation, presuming a steady state condition of flow. Pritchard (2005) developed asymptotic solutions for passive suspended sediment transport under a flood surge on a uniform slope; however, the work was based on a kinematic wave equation. Therefore, the need for analytical and approximate solutions of sediment-laden dam-break flows in general conditions is obvious.

This paper aims to derive approximate solutions for ideal dam-break sediment-laden flows resulting from the sudden release of a finite volume of frictionless, incompressible watersediment mixture on a uniform slope as opposed to a horizontal bed presumed by Pritchard and Hogg (2002). On an inclined bed without friction, the flow will accelerate unboundedly as in previous studies (e.g., Ancey et al. 2008; Fernandez-Feria, 2006), while on a horizontal bed without friction, the fluid will flow downstream unlimitedly (e.g., Pritchard \& Hogg 2002; Ritter, 
1892; Stoker, 1957). On the other hand, the absence of basal friction may substantially affect the front dynamics (Ancey et al., 2006; Hogg \& Pritchard, 2004). Therefore, both cases are physically unrealistic and the analytical and approximate solutions are not directly applicable to engineering practice. However, analytical and approximate solutions of both cases facilitate benchmark solutions, especially when model developers and end-users are still extremely keen to have them to test numerical models. The present work is based on a simplified version of the full set of SHSM equations (Cao et al., 2017), which includes mass and momentum conservation equations for the water-sediment mixture flow, and a mass conservation equation for sediment. Here it is assumed that sediment particles are uniform in size, bed deformation is negligible, and the effects of sediment transport on the mixture flow can be ignored. Notwithstanding that certain mechanisms are overlooked, the governing equations here are more comprehensive physically than previously considered, representing non-equilibrium rather than equilibrium sediment transport (Fraccarollo \& Capart, 2002), adopting a dynamic instead of kinematic wave equation for the mixture flow (Pritchard, 2005), and taking into account unsteady other than steady state condition of flow (Berthon et al., 2012). Succinctly, the present work extends Fernandez-Feria (2006) for clear-water dam-break flows to sediment-laden dam-break flows, and Pritchard and Hogg (2002) for sediment-laden flows over a horizontal bed to an inclined bed. It is significant for verifying numerical SHSM models, as some numerical methods excessively smear waves associated with sediment continuity equation, resulting in rather inaccurate or even incorrect predictions (Toro, 2001). Nevertheless, it is noted that the present work is approximate as parts of the solutions demand numerical realization.

This paper is organized as follows. Section 2 presents the governing equations. In section 3 , the solution strategy of Fernandez-Feria (2006) for ideal dam-break flow (without sediment transport) is applied to derive approximate solutions for the water-sediment mixture flow, while sediment concentration evolution is solved based on the ordinary differential equation (ODE) along its characteristic curve. Section 4 outlines the numerical scheme based on FVM. Section 5 presents a comparison between the approximate solutions and numerical predictions for three distinct sediment transport scenarios: pure advection; pure sedimentation; and concurrent entrainment and deposition. Finally, conclusions are drawn in section 6.

\section{Governing Equations}

In order that the SHSM equations can be tractable to obtain approximate solutions, it is necessary to invoke several simplifications (Ancey et al., 2008; Fernandez-Feria, 2006; Fraccarollo \& Capart, 2002; Pritchard \& Hogg, 2002). First, the bed is taken to be frictionless. Second, it is assumed that bed deformation and the effects of sediment transport on the mixture flow are negligible, which means the mass conservation equation for bed material together with the sediment-related terms in the momentum equation for the mixture flow are decoupled from the SHSM systems and can be eliminated. As a result, the governing equations for the mixture flow here are identical in form to those for clear water flows. Third, uniform sediment transport is considered, which means sediment particles share the same density, shape and size. Under the aforementioned assumptions, the SHSM equations can be written in a conservative form: 
where $t$ is time; $x$ is the downstream coordinate; $h$ is the flow depth; $u$ is the depth-averaged stream-wise flow velocity; $g$ is gravitational acceleration; $\theta$ is the bed inclining angle; $c$ is the depth-averaged sediment concentration; $E$ represents the sediment entrainment flux; and $D$ represents the sediment deposition flux.

It is noted that the understanding of the mechanism of sediment exchange with the bed remains far from complete to date, which inevitably renders uncertainty in its estimation $(=E$ $D$ ). Comparatively, the uncertainty in the entrainment estimation prevails, whilst the determination of the deposition flux is practically more reliable. Here, the conventional relationships are introduced for the entrainment and deposition fluxes (Cao \& Carling, 2002; Huang et al., 2014; Qain et al., 2015; Wu, 2007; Xia et al., 2010)

$$
\begin{aligned}
& E=\alpha \omega_{0} c_{*} \\
& D=\alpha \omega_{0} c
\end{aligned}
$$

where $\alpha$ is an empirical parameter representing the ratio of the near-bed sediment concentration to the depth-averaged sediment concentration, and is specified to be unity here for simplicity; $\omega_{0}$ denotes the settling velocity of a single sediment particle; and $c_{*}$ denotes the sediment transport capacity determined by local flow conditions. In this connection, the approximate solutions derived here are not limited to specific formulas for $c_{*}$; rather they are intended to be valid for general transport capacity formulas in the context of river dynamics (e.g., Celik \& Rodi, 1991; Dey, 2014; Wu, 2007; Yang, 1996).

Formulated in a Cartesian coordinate system with axes aligned along and perpendicular to the bed, equations (1)-(3) are now recast into the following non-conservative form in terms of primitive variables, ready for the derivation of approximate solutions.

$$
\begin{gathered}
\frac{\partial h}{\partial t}+h \frac{\partial u}{\partial x}+u \frac{\partial h}{\partial x}=0 \\
\frac{\partial u}{\partial t}+u \frac{\partial u}{\partial x}+g \cos \theta \frac{\partial h}{\partial x}=g \sin \theta \\
\frac{\partial c}{\partial t}+u \frac{\partial c}{\partial x}=\frac{\alpha \omega_{0}}{h}\left(c_{*}-c\right)
\end{gathered}
$$




\section{Approximate Solutions}

\subsection{Normalization and Homogenization}

We choose the initial flow depth $H_{0}$ at the dam wall, $\sqrt{g H_{0}}$, and the initial sediment concentration $C_{0}$ at a given location as the length scale, velocity scale, and sediment concentration scale. Then the dimensionless variables are deduced as follows:

$$
T=\frac{t}{\sqrt{H_{0} / g}}, X=\frac{x}{H_{0}}, U=\frac{u}{\sqrt{g H_{0}}}, H=\frac{h}{H_{0}}, C=\frac{c}{C_{0}}, \beta=\frac{\omega_{0}}{\sqrt{g H_{0}}} \text {, and } C_{*}=\frac{c_{*}}{C_{0}}
$$

Substitution of the dimensionless variables (9) into equations (6)-(8) yields the following normalized equations

Initial conditions are given as follows:

$$
\frac{\partial C}{\partial T}+U \frac{\partial C}{\partial X}=\frac{\alpha \beta}{H}\left(C_{*}-C\right)
$$

where $e \equiv \tan \theta$ for convenience; and $F(X)$ is the expression of initial sediment concentration distribution. Figure 1 illustrates the initial configuration dictated by equations (13) and (14).

In order to render the governing equations homogeneous and simplify the calculations, the dimensionless variables are altered to:

$$
\tilde{T}=T, \tilde{X}=X-\frac{1}{2} \sin \theta \cdot T^{2}, \tilde{U}=U-\sin \theta \cdot T, \tilde{\eta}=\sqrt{H \cos \theta}, \tilde{C}=C, \text { and } \tilde{C}_{*}=C_{*}
$$

After transformation, equations (10)-(15) are mapped onto

$$
2 \frac{\partial \tilde{\eta}}{\partial \tilde{T}}+\tilde{\eta} \frac{\partial \tilde{U}}{\partial \tilde{X}}+2 \tilde{U} \frac{\partial \tilde{\eta}}{\partial \tilde{X}}=0
$$


192

193

194

195

196

197

198

199

200

201

202

203

$$
\mathbf{W}=\left[\begin{array}{c}
\tilde{\eta} \\
\tilde{U} \\
\tilde{C}
\end{array}\right], \mathbf{A}=\left[\begin{array}{ccc}
\tilde{U} & \tilde{\eta} / 2 & 0 \\
2 \tilde{\eta} & \tilde{U} & 0 \\
0 & 0 & \tilde{U}
\end{array}\right] \text {, and } \mathbf{R}=\left[\begin{array}{c}
0 \\
0 \\
\alpha \beta \cos \theta \cdot\left(\tilde{C}_{*}-\tilde{C}\right) / \tilde{\eta}^{2}
\end{array}\right]
$$

$$
\frac{\partial \mathbf{W}}{\partial \tilde{T}}+\mathbf{A} \frac{\partial \mathbf{W}}{\partial \tilde{X}}=\mathbf{R}
$$

in which

where $\mathbf{W}$ is the vector of primitive variables; $\mathbf{A}$ is the Jacobian matrix; and $\mathbf{R}$ is the vector of 205 206

207 source terms. According to the method of characteristics, the characteristic curves and the corresponding compatibility equations are given below as:

$$
\left\{\begin{array}{l}
\frac{d \tilde{X}}{d \tilde{T}}=\lambda_{1}=\tilde{U}+\tilde{\eta} \\
\frac{d(\tilde{U}+2 \tilde{\eta})}{d \tilde{T}}=0
\end{array}\right.
$$




$$
\left\{\begin{array}{l}
\frac{d \tilde{X}}{d \tilde{T}}=\lambda_{2}=\tilde{U}-\tilde{\eta} \\
\frac{d(\tilde{U}-2 \tilde{\eta})}{d \tilde{T}}=0
\end{array}\right.
$$

\subsection{Solutions for the Mixture Flow}

As mentioned in section 2, the governing equations (6) and (7) for the mixture flow here are identical in form to those for clear water flow. Hence, the solution strategy of FernandezFeria (2006) for ideal dam-break flow (without sediment transport) is applied herein. Succinctly, the strategy features an integration of the adoption of exact solutions and the method of characteristics. Given that both the left and right fronts of the flood wave are initially singular points, namely $\tilde{X}=-1 / e$ and $\tilde{X}=e$ with $\tilde{\eta}=\tilde{U}=0$, they are unsuitable as starting points for the method of characteristics. Therefore, the exact solutions would be useful to serve as the starting condition near these singular points as well as provide the spreading of the two fronts at the initial stages. In order to adopt the exact solutions, which had explicit expressions originally derived for dam-break flows caused by an initial semi-infinite mass of water (Thacker, 1981), Fernandez-Feria (2006) divided the initial fluid (Figure 2) vertically around the origin into two portions and presumed a semi-infinite fluid behind each respectively. Actually, according to the method of characteristics, the exact solutions are invalid in the domain of influence of the imaginary fluid. Correspondingly, the regions of validity of the exact solutions in space and time are specified near both fronts by the characteristic curves starting at the origin (supporting information Figure S1). Outside these regions, the method of characteristics is employed by virtue of equations (25) and (26).

A brief summary of the explicit exact solutions mentioned above is provided below, with detailed developments available in the supporting information Text S1. The exact solution that expresses the propagation of the left-hand flow front is

$$
\tilde{U}(\tilde{T}, \tilde{X})=-\sin \theta \cdot \tilde{T}
$$

$$
\tilde{\eta}(\tilde{T}, \tilde{X})=\left\{\begin{array}{lr}
0 & \text { if } \tilde{X}<l_{1}(\tilde{T}) \equiv-1 / e-\sin \theta \cdot \tilde{T}^{2} / 2 \\
\sqrt{\sin \theta} \sqrt{\tilde{X}+1 / e+\sin \theta \cdot \tilde{T}^{2} / 2} & \text { if } \tilde{X} \geq l_{1}(\tilde{T})
\end{array}\right.
$$

where $l_{1}(\tilde{T})$ represents the trajectory of the left front. The corresponding characteristic curve starting at the point $\tilde{X}=0$ for $\widetilde{T}=0$ is given by

$$
A_{1}(\tilde{T})=-\sqrt{\cos \theta} \cdot \tilde{T}-\sin \theta \cdot \tilde{T}^{2} / 4
$$


237 This determines the region of the plane $(\tilde{X}, \tilde{T})$ corresponding to the range of validity of the 238 exact solution (28) and (29) as follows

$$
l_{1}(\tilde{T}) \leq \tilde{X} \leq A_{1}(\tilde{T})
$$

within which the left-hand flow is independent of any influence from the right portion of the initial fluid before the instant

$$
\tilde{T}_{1} \equiv 2 \frac{\sqrt{\cos \theta}}{\sin \theta}
$$

at which the curves $l_{1}(\tilde{T})$ and $A_{1}(\tilde{T})$ cross each other.

Likewise, the initial stage of the advance of the right flow front is given by

where $l_{2}(\tilde{T})$ represents the trajectory of the right front. The corresponding characteristic curve starting at the point $\tilde{X}=0$ is expressed by

$$
A_{2}(\tilde{T})=\sqrt{\cos \theta} \cdot \tilde{T}+\frac{\cos \theta}{e} \cdot \frac{\tilde{T}^{2}}{4}
$$

which now determines the region of the plane $(\tilde{X}, \tilde{T})$ where the exact solution (33) and (34) remains valid for the right flow front without any influence from the left portion of the initial fluid. Thus, the exact solution (33) and (34) is valid on the right hand side of the origin point over the range

$$
A_{2}(\tilde{T}) \leq \tilde{X} \leq l_{2}(\tilde{T})
$$

until the time

$$
\tilde{T}_{2} \equiv 2 \frac{e}{\sqrt{\cos \theta}}
$$

at which the curves $l_{2}(\tilde{T})$ and $A_{2}(\tilde{T})$ cross each other.

59

60

261

62

\footnotetext{
the right hand side, and has a general solution (supporting information Text S2, Figures S2 and S3)
}

\subsection{Solutions for Sediment Transport}

The sediment transport compatibility equation (27b) is an ODE with a non-zero term on 


$$
\begin{aligned}
\tilde{C}-\tilde{C}_{*} & =-Y_{1}^{-1} \cdot\left(\int \frac{d \tilde{C}_{*}}{d \tilde{T}} \cdot Y_{1} d \tilde{T}+M\right) \\
Y_{1} & =\exp \left(\int \frac{\alpha \beta \cdot \cos \theta}{\tilde{\eta}^{2}} d \tilde{T}\right)
\end{aligned}
$$

where $M$ is an integral constant. Note that $\tilde{C}_{*}$ is determined by the local flow conditions and requires update in each time step. The lower limit of the integrals in equation (38) is set to $\tilde{T}=\tilde{T}_{0}$ when the initial sediment concentration $\widetilde{C}=\widetilde{C}_{0}$ and the initial sediment transport capacity $\tilde{C}_{*}=\tilde{C}_{* 0}$. Setting the upper limit of the integrals to $\tilde{T}=\tilde{T}_{1}$, the particular solution is given by

$$
\tilde{C}=\tilde{Z}_{1}+\tilde{Z}_{2}+\tilde{Z}_{3}
$$

$$
\tilde{Z}_{1}=\widetilde{C}_{*}
$$

$$
\tilde{Z}_{3}=-Y_{2}^{-1} \cdot \int_{\tilde{T}_{0}}^{\tilde{T}_{1}}\left(\frac{d \tilde{C}_{*}}{d \tilde{T}} \cdot Y_{3}\right) d \tilde{T}, \quad Y_{3}=\exp \left(\int_{\tilde{T}_{0}}^{\tilde{T}} \frac{\alpha \beta \cdot \cos \theta}{\tilde{\eta}^{2}} d \tilde{T}^{\prime}\right)
$$

\subsection{Numerical Realization}

Although analytical solutions (25) and (26) are not fully explicit expressions, they can be manipulated numerically readily by utilizing the method of characteristics as equations (25b) and (26b) are homogeneous. On the other hand, the solution (39) for the evolution of sediment concentration field involves integrals, which also require numerical realization. This is why the solutions presented in this paper are approximate.

Briefly, the exact solutions (28) and (29) are adopted in the region prescribed by equation (31) until the time instant expressed by equation (32), and the exact solutions (33) and (34) are employed in the region dictated by equation (36) until the time instant given by equation (37). Over the remaining region of space and time, the method of characteristics is deployed to resolve the mixture flow according to equations (25) and (26), with a predictor-corrector method and a parabolic interpolation to achieve second-order accuracy in both time and space. Meanwhile, the sediment concentration along the characteristic curve is predicted by equation (39), with the integrations evaluated using the trapezoidal rule. Following mesh independence tests, a time step $\Delta \widetilde{T}=0.005$ and a spatial step $\Delta \widetilde{X}=0.002$ are selected.

\section{Finite Volume Method}

The governing equations (1)-(3) are simplified from the full SHSM equations (Cao et al., 2017). Numerical solutions of these equations can be readily attained using a scheme based on 
FVM, given the plenty of computational studies for a hierarchy of complicated flow and sediment transport processes over erodible beds as stated by Cao et al. (2017). The numerical solutions can then be compared with the approximate solutions given above.

Equations (1)-(3) are rearranged in a hyperbolic vector-matrix form as

in which

$$
\frac{\partial \mathbf{V}}{\partial t}+\frac{\partial \mathbf{G}}{\partial x}=\mathbf{S}
$$

where $\mathbf{V}$ is the vector of conservative variables; $\mathbf{G}$ is the vector of fluxes; and $\mathbf{S}$ is the vector of source terms. Under the framework of FVM, an explicit discretization of equation (40) gives

$$
\begin{gathered}
\mathbf{V}_{i}^{p}=\mathbf{V}_{i}^{n}-\frac{\Delta t}{\Delta x}\left[\mathbf{G}_{i+1 / 2}-\mathbf{G}_{i-1 / 2}\right]^{n} \\
\mathbf{V}_{i}^{n+1}=\mathbf{V}_{i}^{p}+\Delta t \cdot \mathbf{S}^{R K}\left(\mathbf{V}_{i}\right)
\end{gathered}
$$

where the superscript $n$ denotes the time step index; the subscript $i$ denotes the spatial node index; $\Delta t$ is the time step; $\Delta x$ is the spatial increment; $\mathbf{G}_{i+1 / 2}$ and $\mathbf{G}_{i-1 / 2}$ represent the inter-cell fluxes; and $\mathbf{S}^{R K}$ represents the source terms which are solved by the second-order Runge-Kutta method as follows:

$$
\begin{gathered}
\mathbf{S}^{R K}\left(\mathbf{V}_{i}\right)=\frac{1}{2}\left[\mathbf{S}\left(\mathbf{V}_{i}^{p}\right)+\mathbf{S}\left(\mathbf{V}_{i}^{*}\right)\right] \\
\mathbf{V}_{i}^{*}=\mathbf{V}_{i}^{p}+\Delta t \cdot \mathbf{S}\left(\mathbf{V}_{i}^{p}\right)
\end{gathered}
$$

The inter-cell fluxes are calculated by the Slope-Limiter Centered (SLIC) method, in which the total variation diminishing (TVD) version of the Monotonic Upstream-Centered Scheme for Conservation Laws (MUSCL) is employed for data reconstruction. Accordingly, numerical predictions here achieve second-order accuracy in both space and time (Toro, 2001).

The spatial step is set to $\Delta x=0.01$, satisfying the criteria of mesh independence. Numerical stability is controlled by the Courant-Friedrichs-Lewy (CFL) criterion (Toro, 2001)

$$
\begin{gathered}
\Delta t=\frac{\Delta x}{\max \left(\left|u_{i}\right|+\sqrt{g h_{i}}\right)} \cdot C r \\
0<C r \leq 1.0
\end{gathered}
$$

where $\mathrm{Cr}$ represents the Courant number and a value of 0.95 is adopted. 


\section{Results}

This study is primarily concerned with the evolution of the sediment concentration field in shallow water-sediment flow over a bed with uniform slope. Three typical sediment transport scenarios are considered, i.e., pure advection; pure sedimentation; and concurrent entrainment and deposition. Although an accelerating, high velocity dam-break flow would be likely to produce substantial sediment entrainment as time progresses, the essentially academic cases selected here examine different forms of the source terms in the sediment continuity equation that relate to key physical mechanisms (see Table 1) and are very useful for model verification.

While sediment transport capacity can be calculated by one of a plethora of formulas in general (Celik \& Rodi, 1991; Dey, 2014; Wu, 2007; Yang, 1996), a specific formula is demanded here to test the proposed approximate solutions. The Zhang formula is based on the theory of energy balance along with laboratory flume and field observed datasets and has seen wide applications in engineering practice (Guo, 2002; Wu, 2007; Zhang \& Xie, 1993). By virtue of logarithmic matching method, Guo (2002) gave an explicit expression of the Zhang formula as follows

$$
c_{*}=\gamma \frac{1}{20 \rho_{s}} \frac{\left[u^{3} / g h \omega_{0}\right]^{1.5}}{1+\left[u^{3} / 45 g h \omega_{0}\right]^{1.15}}
$$

where $\gamma=0.1$ is a modification coefficient introduced herein to prevent the calculated sediment transport capacity from being excessively large (e.g. for high velocity dam-break flows over a frictionless bed with steep slope); and $\rho_{s}$ denotes sediment density. Notwithstanding that the Zhang formula applies to suspended load (Guo, 2002; Wu, 2007; Zhang \& Xie, 1993), alternative formulas for either bed load or total bed-material load can be employed instead, for which the present approximate solutions continue to hold.

The study parameters are as follows. The bed slope has angle $\theta=30^{\circ}$. The sediment particle diameter is $d=0.2 \mathrm{~mm}$ with a dimensionless settling velocity of about $\beta=0.006$ according to the formula of Zhang and Xie (1993). The density of sediment is $\rho_{s}=2650 \mathrm{~kg} / \mathrm{m}^{3}$. In each scenario, two distinct initial sediment distributions are considered: one is a uniform distribution such that

$$
F(X)=1
$$

and the other a linear distribution such that

$$
F(X)=\frac{e X+1}{e^{2}+1} \times 0.9+0.1
$$

Note that $F(\tilde{X})=F(X)$ initially. The scaled sediment concentration is set to $C_{0}=0.01$. For convenience, the approximate solutions and the FVM numerical predictions presented in Figures 3-18 are all in terms of dimensionless variables. 


\subsection{Mixture Flow}

Obviously, since bed deformation and the effects of sediment transport on the mixture flow are neglected, all the cases mentioned above share the same solutions for the mixture flow. Figure 3 shows the approximate solutions and corresponding numerical predictions by FVM for the advancing flow fronts with time. Note that the left front remains at rest until the backward wave reaches the upstream boundary. Figure 4 illustrates the approximate solutions and FVM numerical predictions for the mixture flow at four instants of time. Figure 4 a shows the gradual attenuation of the mixture flow with time, which is consistent with Figure $4 \mathrm{~b}$ where it can be observed that the downstream portion of flow consistently propagates faster than the upstream portion. Moreover, the flow velocity appears to be increased progressively along the domain with time, in keeping with mass conservation (Figure 4). Apparently, the numerical predictions are indistinguishable from the approximate solutions (Figures 3 and 4). On the one hand, the FVM velocity profiles manifest sudden steps at the beginning and end of the flow regions in keeping with the shock-capturing capability of the FVM. On the other hand, the results underline the significance of the present approximate solutions for verifying SHSM models.

\subsection{Pure Advection}

Here sediment transport is assumed to be in an equilibrium state, such that $E=D=0$. Now the corresponding governing equations can also describe the pure advection of a passive scalar, such as the concentration of solute pollutant, neutrally buoyant particles or wash load that barely exchanges with the bottom boundary (Dey, 2014; Yang, 1996; Zhang \& Xie, 1993). Accordingly, the particular solution of the ODE (27b) along the characteristic curve (27a) is

$$
\tilde{C}=\widetilde{C}_{0}
$$

It is easy to infer from equation (49) that the sediment concentration remains constant along the characteristic curve. Specifically, the sediment concentration profiles become stretched in time and space with invariable extreme values compared to the initial profile. Results for two different initial sediment distributions are discussed separately below.

Figure 5 shows the approximate solutions obtained for the evolution of sediment concentration field in $X-T$ space. In accordance with the uniformity of the initial distribution, the magnitude of sediment concentration remains constant. Figure 6 compares the approximate solutions and numerical predictions by FVM for sediment transport at four instants of time. These two solutions match rather well with each other and illustrate the stretching of the concentration profiles with time, which demonstrates the ability of the FVM adopted here to capture contact discontinuity in sediment transport.

Figure 7 illustrates the approximate solutions for the evolution of an initially linear sediment concentration field in $X-T$ space under pure advection. The sediment concentration field sustains an almost linear distribution throughout the flow region with the extreme values unaltered. Figure 8 shows the remarkable agreement between the approximate solutions and FVM numerical predictions of the sediment concentration profiles at four instants of time. The 
results again demonstrate the remarkable ability of the FVM to capture discontinuity in sediment transport and also the usefulness of the approximate solutions for model verification purposes.

\subsection{Pure Sedimentation}

Absolute deposition of sediment is now assumed throughout the flow region, without any entrainment from the bed (such that $E=0$ ). Hence, the particular solution (39) for sediment concentration along the characteristic curve can be simplified to give

$$
\tilde{C}=\tilde{C}_{0} \cdot Y_{2}^{-1}, \quad Y_{2}=\exp \left(\int_{\widetilde{T}_{0}}^{\widetilde{T}_{1}} \frac{\alpha \beta \cdot \cos \theta}{\tilde{\eta}^{2}} d \tilde{T}\right)
$$

In light of equation (50), the sediment concentration is expected to attenuate along the characteristic curve. Results obtained for uniform and linear initial sediment distributions are presented below.

Figure 9 shows the approximate solutions obtained for the evolution of an initially uniform distribution of sediment in $X-T$ space. As expected, the concentration profiles diminish with time as sedimentation occurs. Figure 10 compares the approximate solutions and FVM numerical predictions of the sediment concentration profiles at four different times. Again, the numerical predictions reproduce the approximate solutions accurately. It can be seen that the initial profile flattens out and almost disappears by $T=30$.

Figure 11 illustrates the approximate solutions obtained for an initially linear distribution of sediment as it evolves in $X-T$ space under pure sedimentation. The sediment concentration profiles again exhibit a progressive decline with time and eventually wash out as sediment is deposited, the process being completed by $T=30$. Figure 12 confirms the close agreement between the approximate solutions and FVM numerical predictions of the sediment concentration profiles at four different times.

\subsection{Concurrent Entrainment and Deposition}

In general, entrainment and deposition take place synchronously in fluvial processes, which are closer to practice than the scenarios evaluated above (pure advection and sedimentation). Yet, since the flow depth is contained in the denominators of the Zhang formula (46), the sediment transport capacity so calculated would become numerically excessively large at both left and right fronts featuring small flow depths. For numerical considerations, a constraint is introduced herein: sediment transport capacity is set to zero if the dimensionless flow depth is below a sufficiently small value of 0.001 . Results obtained for the two types of initial sediment distribution are presented below.

Figure 13 shows the evolution of sediment concentration field in $X$ - $T$ space for the initially uniform distribution of sediment undergoing both deposition and entrainment. There is a monotonic growth in sediment concentration with peaks at the ends and a depression in between, 
which becomes increasingly evident with time. There is almost perfect agreement between the approximate solutions and FVM predictions as shown in Figure 14.

Figure 15 shows the approximate solutions obtained for the sediment concentration field in $X-T$ space commencing from an initially linear distribution of sediment. Again, there is a monotonic increase in sediment concentration with time, and the end profiles are qualitatively very similar to those obtained for the initially uniform distribution, indicating that the exact shape of the initial profile is unimportant when simultaneous entrainment and deposition are occurring. Close agreement is again obtained between the approximate and numerically predicted sediment concentration profiles in Figure 16.

At first glance, the upward concavity of the sediment concentration profiles in Figures 13-16 may be attributed to the use of the Zhang formula (46), as a smaller flow depth would lead to a higher sediment transport capacity. To clarify, an alternative formula is applied in lieu of the Zhang formula (46). Following Bohorquez and Fernandez-Feria (2008) and Pritchard and Hogg (2002), we consider the following form for the entrainment flux, replacing equations (4) and (46):

$$
E=\left\{\begin{array}{cl}
\frac{m_{e}}{\rho_{s}}\left(\frac{u^{2}}{u_{e}{ }^{2}}-1\right)^{q} & \text { for }|u| \geq u_{e} \\
0 & \text { for }|u|<u_{e}
\end{array}\right.
$$

Apparently, sediment transport capacity $c_{*}$ can be expressed by $E / \alpha \omega_{0}$ as per equation (4). Equation (51) is often used to estimate sediment entrainment rate and no sediment will move until some critical shear stress, corresponding to a velocity $u_{e}$, is exceeded (Dyer \& Soulsby, 1988; Pritchard \& Hogg, 2002; Sanford \& Maa, 2001; Teisson et al., 1993). The quantity $m_{e}$ represents a constant mass flux per unit area that usually ranges between $5 \times 10^{-5}$ and $5 \times 10^{-3} \mathrm{~kg} \cdot \mathrm{m}^{-2} \mathrm{~s}^{-1}$, and a value of $5 \times 10^{-4} \mathrm{~kg} \cdot \mathrm{m}^{-2} \mathrm{~s}^{-1}$ is specified here; $q$ is a dimensionless exponent that is often taken to be unity; and $u_{e}$ denotes the critical velocity for incipient motion of sediment particles and is given by

$$
u_{e}=k \cdot \omega_{0} \sqrt{8 / f}
$$

where $k$ is a dimensionless constant, and a value of 1.2 is specified; $f$ is the Darcy-Weisbach friction factor and a value of 0.03 is utilized. Since the denominators in equation (51) do not contain the flow depth, the aforementioned constraint as per small flow depth is no longer demanded.

Figure 17 shows the corresponding sediment concentration profiles for an initially uniform distribution of sediment. Obviously, upward concave curves are obtained qualitatively similar to those in Figures 13 and 14, and also there is satisfactory agreement between the approximate solutions and the FVM numerical predictions. It follows that the upward concave sediment concentration profiles do not result from the specific sediment transport capacity or entrainment formulations. 


\subsection{Decomposition of Concentration Field}

In order to explain the upward concavity of the sediment concentration profiles, the constituents determining sediment concentration distribution are elaborated. As per equation (39), the sediment concentration at the present time level consists of three parts: $\widetilde{Z}_{1}$ represents the sediment transport capacity at the present time level; $\tilde{Z}_{2}$ means the difference between sediment concentration and sediment transport capacity at the previous time level as modified by the flow conditions; and $\tilde{Z}_{3}$ indicates the deviation in sediment transport capacity due to the change of flow conditions. This is in accordance with the physical mechanisms incorporated here in the mass conservation equation (3) for sediment. Sediment transport tends to be at its capacity in line with the local flow scenario due to the upward entrainment process. On the other hand, sediment transport also involves streamwise advection and downward deposition (gravitational action) processes. In this connection, the sediment concentration field would deviate from its capacity state and be modified corresponding to the local flow and sediment conditions.

Figure 18 shows the sediment concentration profiles and the distributions of $Z_{1}, Z_{2}$, and $Z_{3}$ for an initially uniform distribution of sediment concentration, with the sediment transport capacity evaluated using formulas (46) and (51), respectively. It can be seen from Figure 18 that the sediment concentration $C$ is mainly determined by $Z_{1}$ and $Z_{2}$ since the value of $Z_{3}$ is around zero, which means the deviation in sediment transport capacity along the characteristic curve (27a) is very small. Comparison between either Figures 18a and 18c or Figures 18b and $18 \mathrm{~d}$ demonstrates that the distributions of sediment transport capacity $\left(Z_{1}=C_{*}\right)$ vary significantly owing to the distinct formulas adopted, while all the profiles of $Z_{2}$ are rather similar in curvature. This is because the term $Y_{2}$ in equation (39c) for $Z_{2}$ is determined by the flow depth as $H=\tilde{\eta}^{2} / \cos \theta$ according to equation (16). Consequently, the upward concavity characterizes the distribution of $Z_{2}$ while the flow depth profiles are concave downwards (Figure 4a). Finally, the superposition of the profiles of $Z_{1}$ and $Z_{2}$ leads to the upward concave curves of sediment concentration in Figures 13-17.

Bohorquez and Fernandez-Feria (2008) investigated sediment transport under dam-break flows on a fixed, rough and inclined bed. Their results featured an initial increase and subsequent depression in velocity as well as the formation of roll waves in the long term. Moreover, the recent work by Cao et al. (2015) demonstrates that the turbulent Reynolds stress, expressed by a second-order term in the shallow water equations, plays a considerable role in roll waves modeling. Unfortunately, it remains unrealistic to derive analytical solutions if bed resistance and the second-order term for the Reynolds stress are incorporated. Nevertheless, with bed resistance accounted for, the present FVM model can readily solve the governing equations in Bohorquez and Fernandez-Feria (2008) (supporting information Text S3). Notably, the FVM numerical predictions are consistent with Bohorquez and Fernandez-Feria (2008), in which the sediment concentration profiles do not feature upward concavity as opposed to those in Figures 13-17 (supporting information Figures S4 and S5). Therefore, the upward concave sediment concentration profiles in Figures 13-17 are essentially dictated by the particular flow and sediment conditions, rather than the specific sediment transport capacity or entrainment formulations. 


\section{Conclusions}

Approximate solutions have been derived for ideal dam-break sediment-laden flows initiated by the sudden release of a finite volume of frictionless, incompressible water-sediment mixture on a uniform slope. In order that the reduced, governing SHSM equations are tractable for approximate solutions, it is assumed that sediment transport is uniform and does not affect the mixture flow, and there is negligible bed deformation. The solution strategy of FernandezFeria (2006) for ideal dam-break flow (without sediment transport) is used to resolve the mixture flow, while the sediment concentration evolution is solved based on the compatibility equation along its characteristic curve.

Quantitative results are given for a water-sediment dam-break flow over a bed sloping at an angle of $\theta=30^{\circ}$. The mixture flow profiles feature a gradual depression and expansion accompanied by a persistent increase in velocity. The approximate solutions for sediment transport are the highlights of this work. Under the case of pure advection, the sediment concentration remains constant along the characteristic curve. Under the case of pure sedimentation, the sediment concentration profiles diminish progressively with time and eventually disappear when all sediment in the water column has been deposited on the bed. In the case of concurrent entrainment and deposition, upward concavity characterizes the sediment concentration profiles irrespective of the formulas adopted for sediment transport capacity. This is due to the particular flow and sediment conditions resolved here by virtue of the simplified SHSM equations (1)-(3). In all cases, very close agreement is achieved between the approximate solutions and the FVM numerical predictions, confirming the reliability of FVM model and the potential of the present approximate solutions for testing the ability of SHSM models to capture contact discontinuities in sediment transport.

Extensions of the present work are warranted, albeit challenging. Notably, friction and bed evolution need to be incorporated, non-uniform sediment transport to be accounted for, and the interactions between flow, sediment transport and morphological evolution accommodated so that analytical models contain as nearly a complete representation of the mechanisms of fluvial processes as possible, thereby facilitating minimization of model uncertainty.

\section{Acknowledgments and Data}

The work reported in this paper is funded by the Natural Science Foundation of China (Grant Nos. 11432015 and 51279144). The detailed developments involved in section 3 and the supplementary computations for section 5 are provided in the supporting information. All the data shown in Figures 3-18 of this paper are available through doi.org/10.5281/zenodo.1183446. The constructive suggestions of the anonymous reviewers and the editors are gratefully acknowledged. 


\section{References}

Ancey, C., Cochard, S., Wiederseiner, S., \& Rentschler, M. (2006). Front dynamics of supercritical non-Boussinesq gravity currents. Water Resources Research, 42, W08424. doi:10.1029/2005WR004593

Ancey, C., Iverson, R. M., Rentschler, M., \& Denlinger, R. P. (2008). An exact solution for ideal dam-break floods on steep slopes. Water Resources Research, 44(1), W01430. doi:10.1029/2007WR006353

Antuono, M., \& Hogg, A. J. (2009). Run-up and backwash bore formation from dam-break flow on an inclined plane. Journal of Fluid Mechanics, 640, 151-164. doi:10.1017/S0022112009991698

Berthon, C., Cordier, S., Delestre, O., \& Le, M. H. (2012). An analytical solution of the shallow water system coupled to the Exner equation. Comptes Rendus Mathematique, 350(3-4), 183-186. doi:10.1016/j.crma.2012.01.007

Bohorquez, P. \& Fernandez-Feria, R. (2008). Transport of suspended sediment under the dambreak flow on an inclined plane bed of arbitrary slope. Hydrological Processes, 22(14), 2615-2633. doi:10.1002/hyp.6858

Cao, Z., \& Carling, P. A. (2002). Mathematical modelling of alluvial rivers: reality and myth. Part II: special issues. Water \& Maritime Engineering - Proc. Inst. Civil Engrs., 154(4), 297-307. doi:10.1680/maen.154.4.297.38901

Cao, Z., Pender, G., Wallis, S., \& Carling, P. (2004). Computational dam-break hydraulics over erodible sediment bed. Journal of Hydraulic Engineering, 130(7), 689-703. doi:10.1061/(ASCE)0733-9429(2004)130:7(689)

Cao, Z., Hu, P., Hu, K., Pender, G., \& Liu, Q. (2015). Modelling roll waves with shallow water equations and turbulent closure. Journal of Hydraulic Research, 53(2), 161-177. doi:10.1080/00221686.2014.950350

Cao, Z., Xia, C., Pender, G., \& Liu, Q. (2017). Shallow water hydro-sediment-morphodynamics equations for fluvial processes. Journal of Hydraulic Engineering, 143(5), 02517001. doi:10.1061/(ASCE)HY.1943-7900.0001281

Capart, H., \& Young, D. Y. (1998). Formation of a jump by the dam-break wave over a granular bed. Journal of Fluid Mechanics, 372, 165-187. doi:10.1017/S0022112098002250

Celik, I., \& Rodi, W. (1991). Suspended sediment-transport capacity for open channel flow. Journal of Hydraulic Engineering, 117(2), 191-204. doi:10.1061/(ASCE)07339429(1991)117:2(191)

Cristo, C. D., Greco, M., Iervolino, M., Leopardi, A., \& Vacca, A. (2015). Two-dimensional two-phase depth-integrated model for transients over mobile bed. Journal of Hydraulic Engineering, 142(2), 04015043. doi:10.1061/(ASCE)HY.1943-7900.0001024

Dey, S. (2014). Fluvial Hydrodynamics: Hydrodynamic and Sediment Transport Phenomena. Berlin: Springer.

Dressler, R. F. (1952). Hydraulic resistance effect upon the dam-break functions. Journal of Research of the National Bureau of Standards, 49(3), 217-225. 
Dyer, K. R., \& Soulsby, R. L. (1988). Sand transport on the continental shelf. Annual Review of Fluid Mechanics, 20, 295-324. doi:10.1146/annurev.fl.20.010188.001455

Fernandez-Feria, R. (2006). Dam-break flow for arbitrary slopes of the bottom. Journal of Engineering Mathematics, 54(4), 319-331. doi:10.1007/s10665-006-9034-5

Fraccarollo, L. \& Capart, H. (2002). Riemann wave description of erosional dam-break flows. Journal of Fluid Mechanics, 461, 183-228. doi:10.1017/S0022112002008455

Guo, J. (2002). Logarithmic matching and its applications in computational hydraulics and sediment transport. Journal of Hydraulic Research, 40(5), 555-566. doi:10.1080/00221680209499900

Hogg, A. J. (2006). Lock-release gravity currents and dam-break flows. Journal of Fluid Mechanics, 569, 61-87. doi:10.1017/S0022112006002588

Hogg, A. J., \& Pritchard, D. (2004). The effects of hydraulic resistance on dam-break and other shallow inertial flows. Journal of Fluid Mechanics, 501, 179-212. doi:10.1017/S0022112003007468

Hu, P., \& Cao, Z. (2009). Fully coupled mathematical modeling of turbidity currents over erodible bed. Advances in Water Resources, 32(1), 1-15. doi:10.1016/j.advwatres.2008.07.018

Hu, P., Cao, Z., Pender, G., \& Tan, G. (2012). Numerical modelling of turbidity currents in the Xiaolangdi reservoir, Yellow River, China. Journal of Hydrology, 464-465, 41-53. doi:10.1016/j.jhydrol.2012.06.032

Huang, W., Cao, Z., Yue, Z., Pender, G., \& Zhou, J. (2012). Coupled modelling of flood due to natural landslide dam breach. Proceedings of the Institution of Civil Engineers - Water Management, 165(10), 525-542. doi:10.1680/wama.12.00017

Huang, W., Cao, Z., Carling, P., \& Pender, G. (2014). Coupled 2D hydrodynamic and sediment transport modeling of megaflood due to glacier dam-break in Altai Mountain, Southern Siberia. Journal of Mountain Science, 11(6), 1442-1453. doi:10.1007/s11629-014-3032-2

Kim, D. H. (2015). H2D morphodynamic model considering wave, current and sediment interaction. Coastal Engineering, 95, 20-34. doi:10.1016/j.coastaleng.2014.09.006

Kim, J., Ivanov, V. Y., \& Katopodes, N. D. (2013). Modeling erosion and sedimentation coupled with hydrological and overland flow processes at the watershed scale. Water Resources Research, 49(9), 5134-5154. doi:10.1002/wrcr.20373

Li, J., Cao, Z., Pender, G., \& Liu, Q. (2013). A double layer-averaged model for dam-break flows over mobile bed. Journal of Hydraulic Research, 51(5), 518-534. doi:10.1080/00221686.2013.812047

Li, J., Cao, Z., Hu, K., Pender, G., \& Liu, Q. (2017a). A depth-averaged two-phase model for debris flows over fixed beds. International Journal of Sediment Research, in press. doi:10.1016/j.ijsrc.2017.06.003

Li, J., Cao, Z., Hu, K., Pender, G., \& Liu, Q. (2017b). A depth-averaged two-phase model for debris flows over erodible beds. Earth Surface Processes and Landforms, in press. doi:10.1002/esp.4283 
Li, J., Cao, Z., Qian, H., Liu, Q., \& Pender, G. (2017). A depth-averaged two-phase model for fluvial sediment-laden flows. Advances in Water Resources, in press. doi:10.1016/j.advwatres.2017.08.014

Mangeney, A., Heinrich, P., \& Roche, R. (2000). Analytical solution for testing debris avalanche numerical models. Pure and Applied Geophysics, 157(6-8), 1081-1096. doi: $10.1007 / \mathrm{s} 000240050018$

Michoski, C., Dawson, C., Mirabito, C., Kubatko, E. J., Wirasaet, D., \& Westerink, J. J. (2013). Fully coupled methods for multiphase morphodynamics. Advances in Water Resources, 59, 95-110. doi:10.1016/j.advwatres.2013.05.002

Nicholas, A. P., Ashworth, P. J., Sambrook Smith, G. H., \& Sandbach, S. D. (2013). Numerical simulation of bar and island morphodynamics in anabranching megarivers. Journal of Geophysical Research: Earth Surface, 118(4), 2019-2044. doi:10.1002/jgrf.20132

Pritchard, D., \& Hogg, A. J. (2002). On sediment transport under dam-break flow. Journal of Fluid Mechanics, 473, 265-274. doi:10.1017/S0022112002002550

Pritchard, D. (2005). On fine sediment transport by a flood surge. Journal of Fluid Mechanics, 543, 239-248. doi:10.1017/S0022112005006518

Qian, H., Cao, Z., Pender, G., Liu, H., \& Hu, P. (2015). Well-balanced numerical modelling of non-uniform sediment transport in alluvial rivers. International Journal of Sediment Research, 30(2), 117-130. doi:10.1016/j.ijsrc.2015.03.002

Qian, H., Cao, Z., Liu, H., \& Pender, G. (2017). Numerical modeling of alternate bar formation, development and sediment sorting in straight channels. Earth Surface Processes and Landforms, 42(4), 555-574. doi:10.1002/esp.3988

Ritter, A. (1892). Die fortpflanzung de wasserwellen. Zeitschrift Verein Deutscher Ingenieure, 36(33), 947-954.

Sanford, L. P., \& Maa, J. P. Y. (2001). A unified erosion formulation for fine sediments. Marine Geology, 179(1-2), 9-23. doi:10.1016/S0025-3227(01)00201-8

Spinewine, B., \& Capart, H. (2013). Intense bed-load due to a sudden dam-break. Journal of Fluid Mechanics, 731, 579-614. doi:10.1017/jfm.2013.227

Stoker, J. J. (1957). Water Waves. New York: Wiley-Interscience.

Teisson, C., Ockenden, M., Le Hir, P., Kranenburg, C., \& Hamm, L. (1993). Cohesive sediment transport processes. Coastal Engineering, 21(1-3), 129-162. doi:10.1016/03783839(93)90048-D

Tan, W. (1998). Computational Shallow Water Hydrodynamics. Beijing, China: Tsinghua University Press (in Chinese).

Thacker, W. C. (1981). Some exact solutions to the nonlinear shallow-water wave equations. Journal of Fluid Mechanics, 107, 499-508. doi:10.1017/S0022112081001882

Toro, E. F. (2001). Shock-Capturing Methods for Free-Surface Shallow Flows. Chichester, U. K.: Wiley. 
Wang, G., Xia, J., \& Wu, B. (2008). Numerical simulation of longitudinal and lateral channel deformations in the braided reach of the lower Yellow River. Journal of Hydraulic Engineering, 134(8), 1064-1078. doi:10.1061/(ASCE)0733-9429(2008)134:8(1064)

Whitham, G. B. (1955). The effects of hydraulic resistance in the dam-break problem. Proceedings of the Royal Society A, 227(1170), 399-407. doi:10.1098/rspa.1955.0019

Wu, W. (2007). Computational River Dynamics. London, U. K.: Taylor \& Francis.

Wu, W., \& Wang, S. S. (2007). One-dimensional modeling of dam-break flow over movable beds. Journal of Hydraulic Engineering, 133(1), 48-58. doi:10.1061/(ASCE)07339429(2007)133:1(48)

Xia, J., Lin, B., Falconer, R. A., \& Wang, G. (2010). Modeling dam-break flows over mobile beds using a 2D coupled approach. Advances in Water Resources, 33(2), 171-183. doi:10.1016/j.advwatres.2009.11.004

Xiao, H, Young, Y. L., \& Prévost, J. H. (2010). Hydro- and morpho-dynamic modeling of breaking solitary waves over a fine sand beach. Part II: Numerical simulation. Marine Geology, 269(3-4), 119-131. doi:10.1016/j.margeo.2009.12.008

Xie, J. (Ed.) (1990). River Modeling. Beijing, China: China Water and Power Press (in Chinese).

Yang, C. T. (1996). Sediment Transport: Theory and Practice. New York: McGraw-Hill.

Zech, Y., Soares-Frazão, S., \& Van Emelen, S. (2015). Modeling of fast hydraulic transients: issues, challenges, perspectives. La Houille Blanche, (5), 5-15. doi: $10.1051 / \mathrm{hb} / 20150049$

Zhang, R., \& Xie, J. (1993). Sedimentation Research in China: Systematic Selections. Beijing, China: China Water and Power Press.

Zhu, F., \& Dodd, N. (2015). The morphodynamics of a swash event on an erodible beach. Journal of Fluid Mechanics, 762, 110-140. doi:10.1017/jfm.2014.610

Zoppou, C., \& Roberts, S. (2003). Explicit schemes for dam-break simulations. Journal of Hydraulic Engineering, 129(1), 11-34. doi:10.1061/(ASCE)0733-9429(2003)129:1(11) 
Confidential manuscript submitted to Water Resources Research

689 Table 1. Summary of Sediment Transport Scenarios

Scenarios

\begin{tabular}{c|c} 
Scenarios & Source terms in equatio \\
\hline Pure advection & $0(E=D=0)$ \\
Pure sedimentation & $-D$ \\
Concurrent entrainment and deposition & $E-D$
\end{tabular}

690

691 


\section{List of Figure captions}

Figure 1. Initial configuration in terms of dimensionless variables.

Figure 2. Initial configuration in terms of transformed variables.

696

697

698

699

700

701

702

703

704

705

706

707

708

709

710

711

712

713

714

715

716

717

718

719

720

721

722

723

724

Figure 3. Approximate solutions and FVM numerical predictions of the locations of the advancing flow fronts with time.

Figure 4. Approximate solutions and FVM numerical predictions of the water-sediment mixture flow at four instants of time: (a) depth profiles; and (b) velocity profiles. The magenta lines in (b) are plotted outside the flow region to delineate more clearly the approximate solutions obtained for the velocity of the flow fronts.

Figure 5. Approximate solutions for the evolution of sediment concentration field in $X-T$ space for an initially uniform distribution of sediment under pure advection.

Figure 6. Approximate solutions and FVM numerical predictions of the sediment concentration profiles at four instants of time for an initially uniform distribution of sediment under pure advection.

Figure 7. Approximate solutions for the evolution of sediment concentration field in $X$ - $T$ space for an initially linear (triangular) distribution of sediment under pure advection.

Figure 8. Approximate solutions and FVM numerical predictions of the sediment concentration profiles at four instants of time for an initially linear (triangular) distribution of sediment under pure advection.

Figure 9. Approximate solutions for the evolution of sediment concentration field in $X$ - $T$ space for an initially uniform distribution of sediment under pure sedimentation.

Figure 10. Approximate solutions and FVM numerical predictions of the sediment concentration profiles at four instants of time for an initially uniform distribution of sediment under pure sedimentation.

Figure 11. Approximate solutions for the evolution of sediment concentration field in $X$ - $T$ space for an initially linear (triangular) distribution of sediment under pure sedimentation.

Figure 12. Approximate solutions and FVM numerical predictions of the sediment concentration profiles at four instants of time for an initially linear (triangular) distribution of sediment under pure sedimentation.

Figure 13. Approximate solutions for the evolution of sediment concentration field in $X-T$ space for an initially uniform distribution of sediment when entrainment and deposition occur concurrently. 
Figure 14. Approximate solutions and FVM numerical predictions of the sediment concentration profiles at four instants of time for an initially uniform distribution of sediment when entrainment and deposition occur concurrently.

Figure 15. Approximate solutions for the evolution of sediment concentration field in $X$ - $T$ space for an initially linear (triangular) distribution of sediment when entrainment and deposition occur concurrently.

Figure 16. Approximate solutions and FVM numerical predictions of the sediment concentration profiles at four instants of time for an initially linear (triangular) distribution of sediment when entrainment and deposition occur concurrently.

Figure 17. Approximate solutions and FVM numerical predictions of the sediment concentration profiles at four instants of time for an initially uniform distribution of sediment when entrainment and deposition occur concurrently. Sediment transport capacity is calculated according to formula (51).

Figure 18. Sediment concentration profiles along with the distributions of dimensionless values $Z_{1}, Z_{2}$, and $Z_{3}$ in equation (39) at two instants of time for an initially uniform distribution of sediment concentration. Sediment transport capacity is calculated by the Zhang formula (46) in (a) and (b), and by formula (51) in (c) and (d). 
Figure 1. 


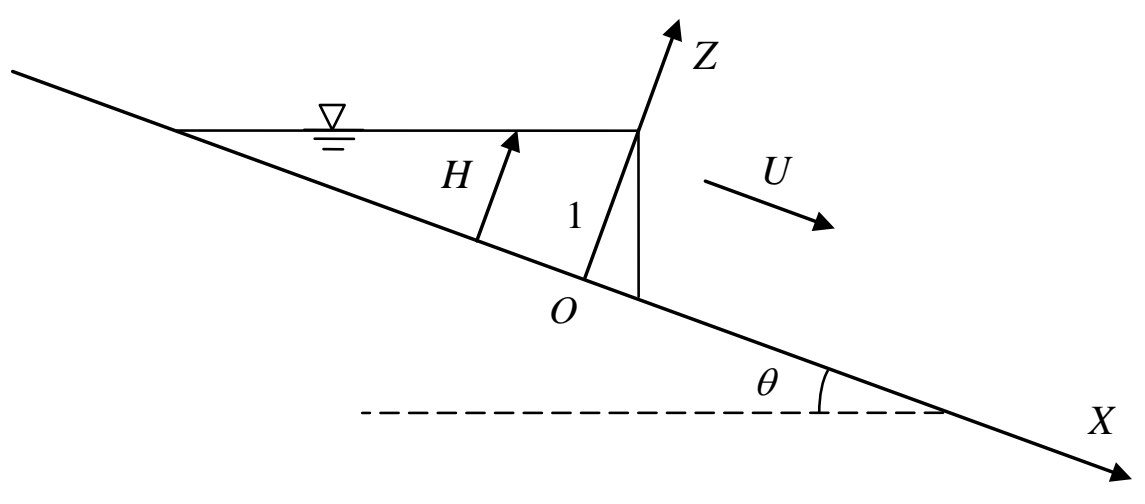


Figure 2. 


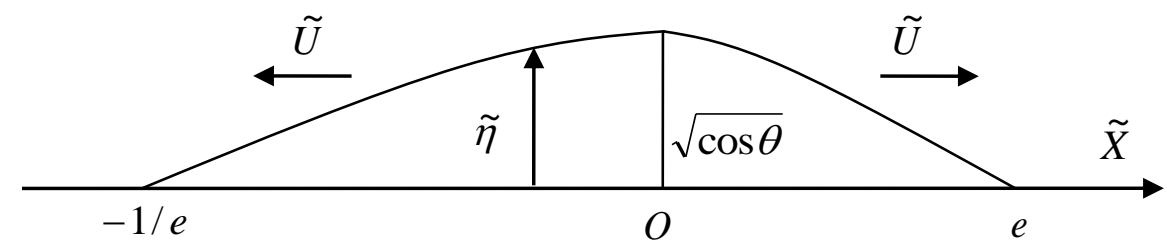


Figure 3. 


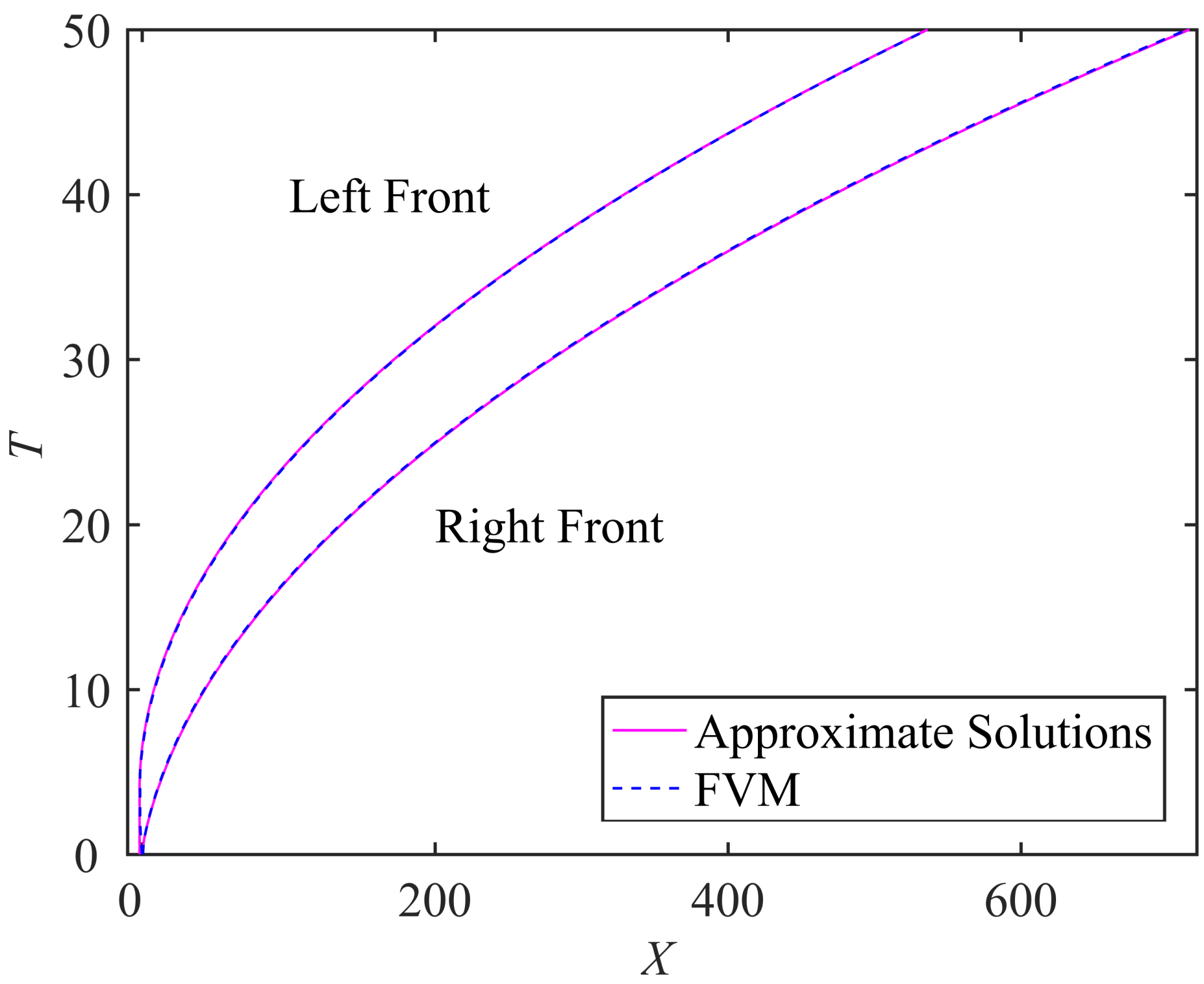


Figure 4. 

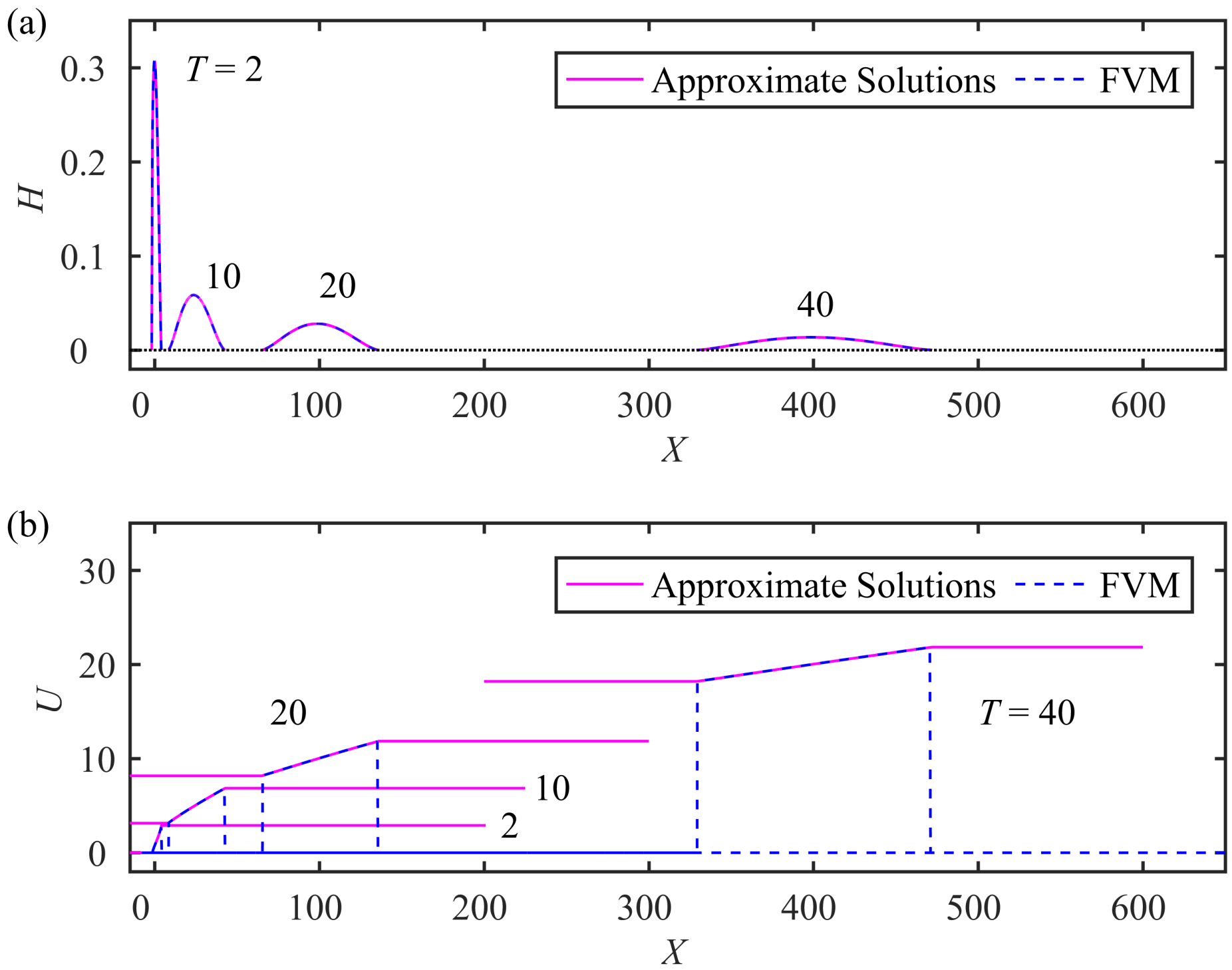
Figure 5. 


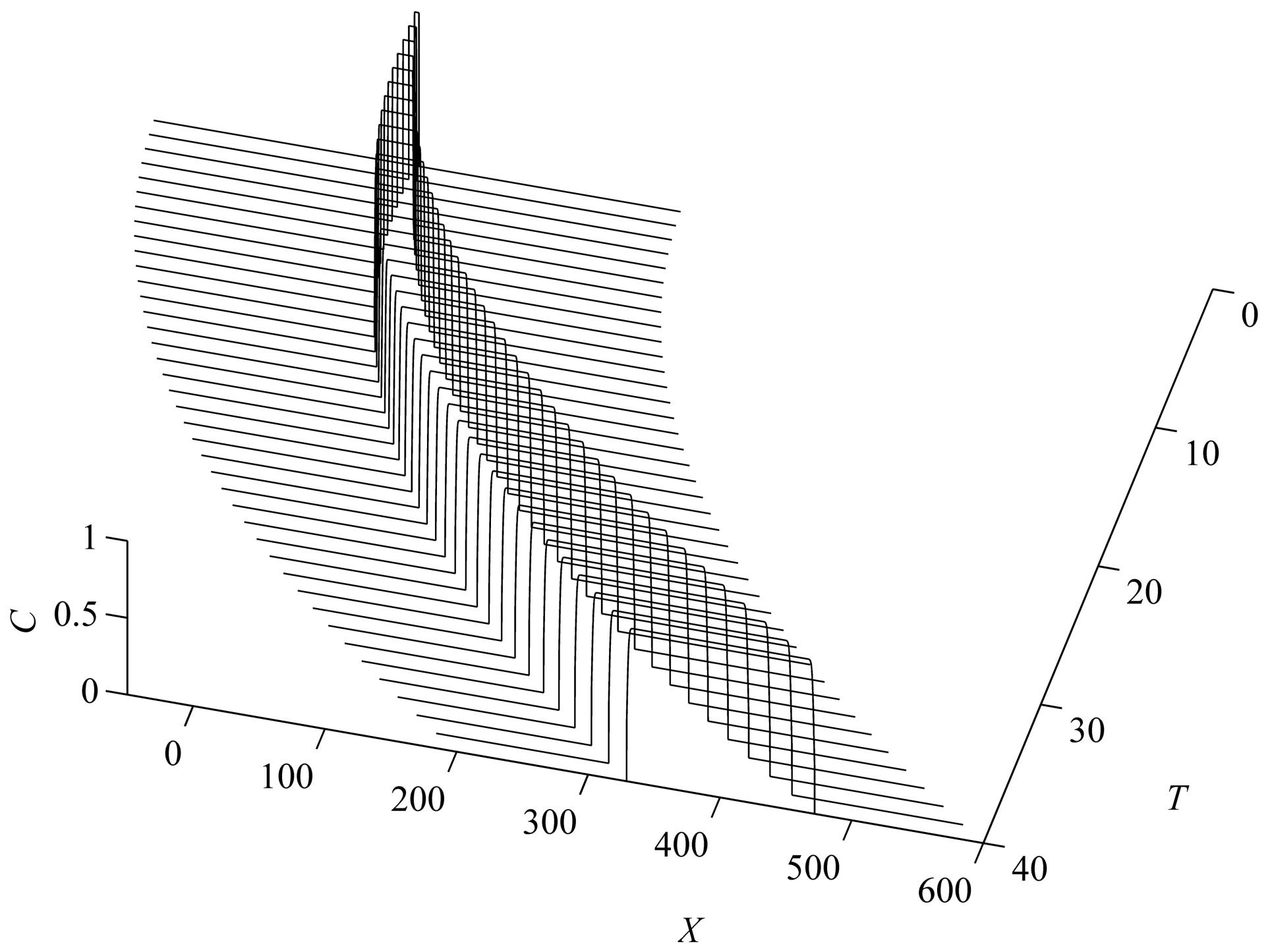


Figure 6. 


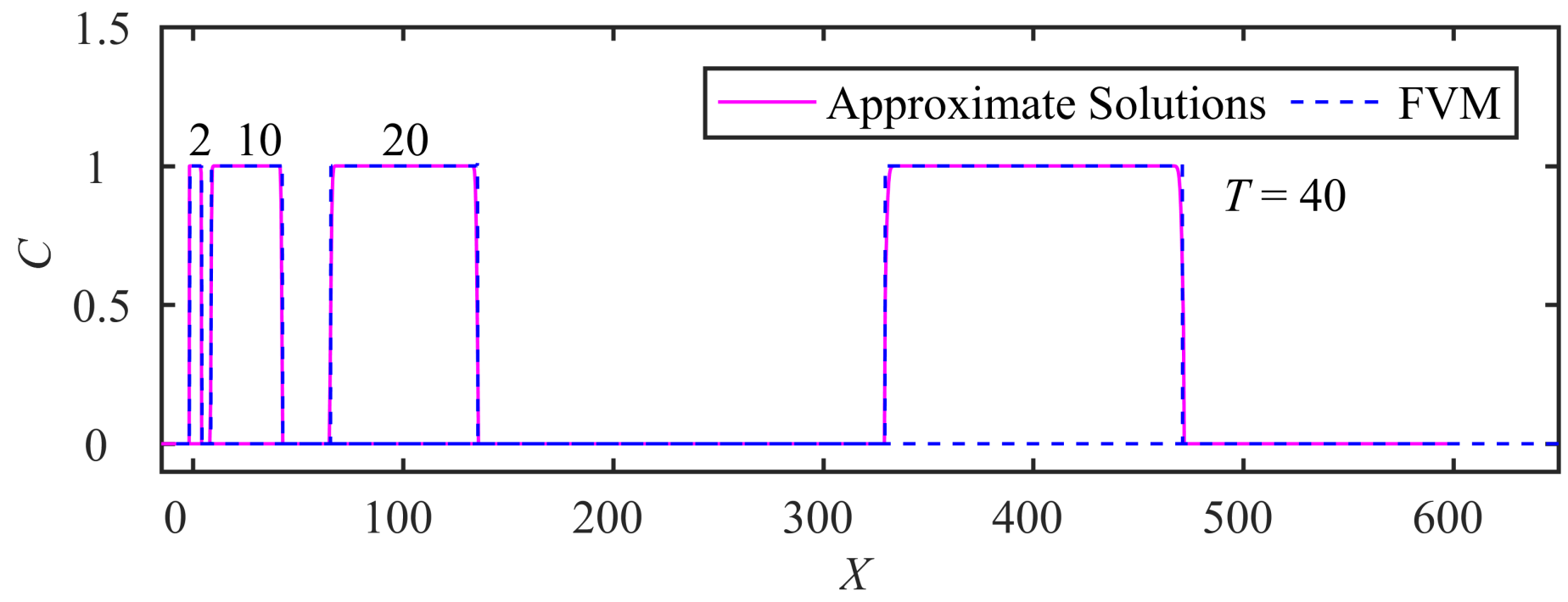


Figure 7. 


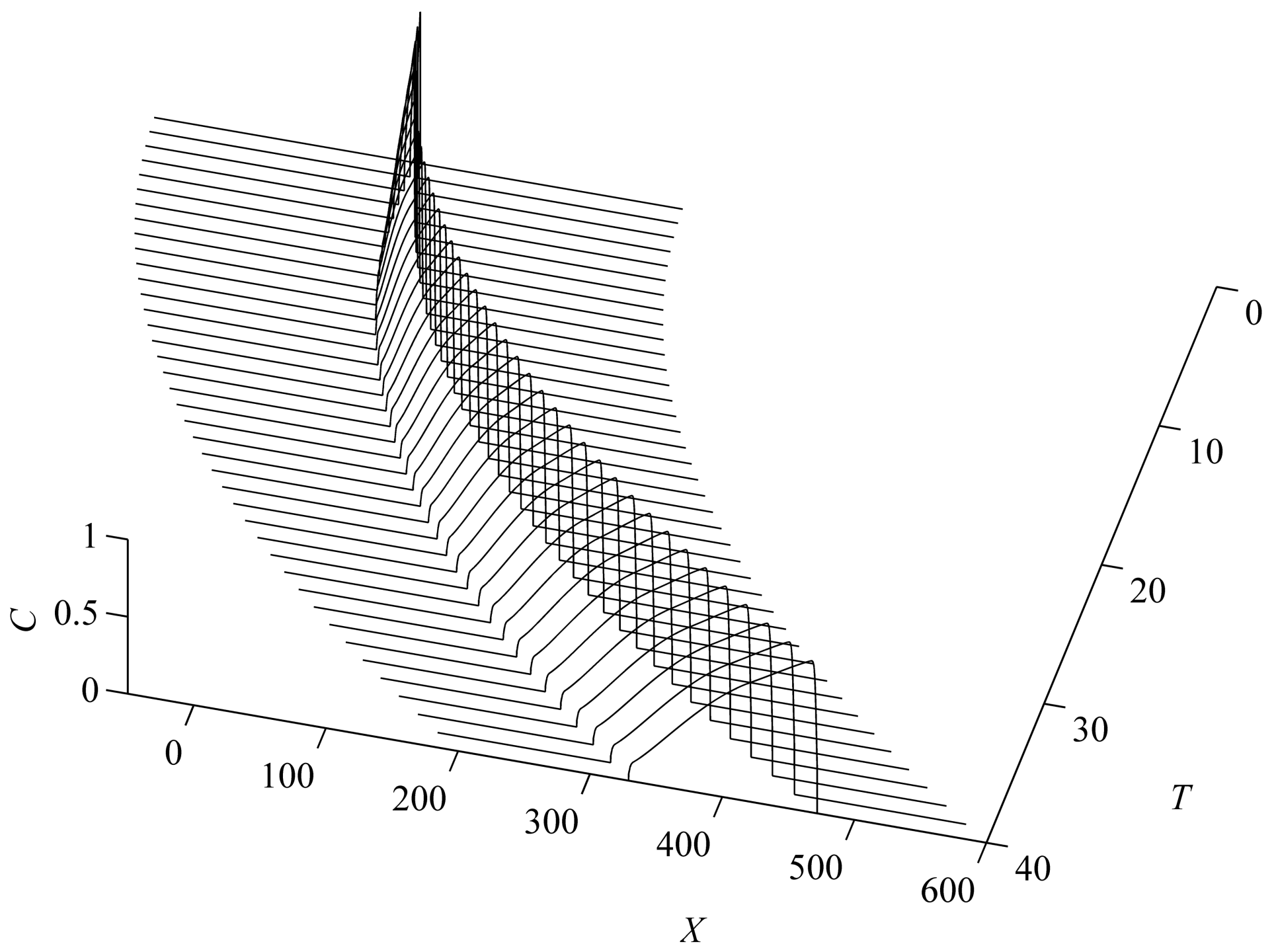


Figure 8. 


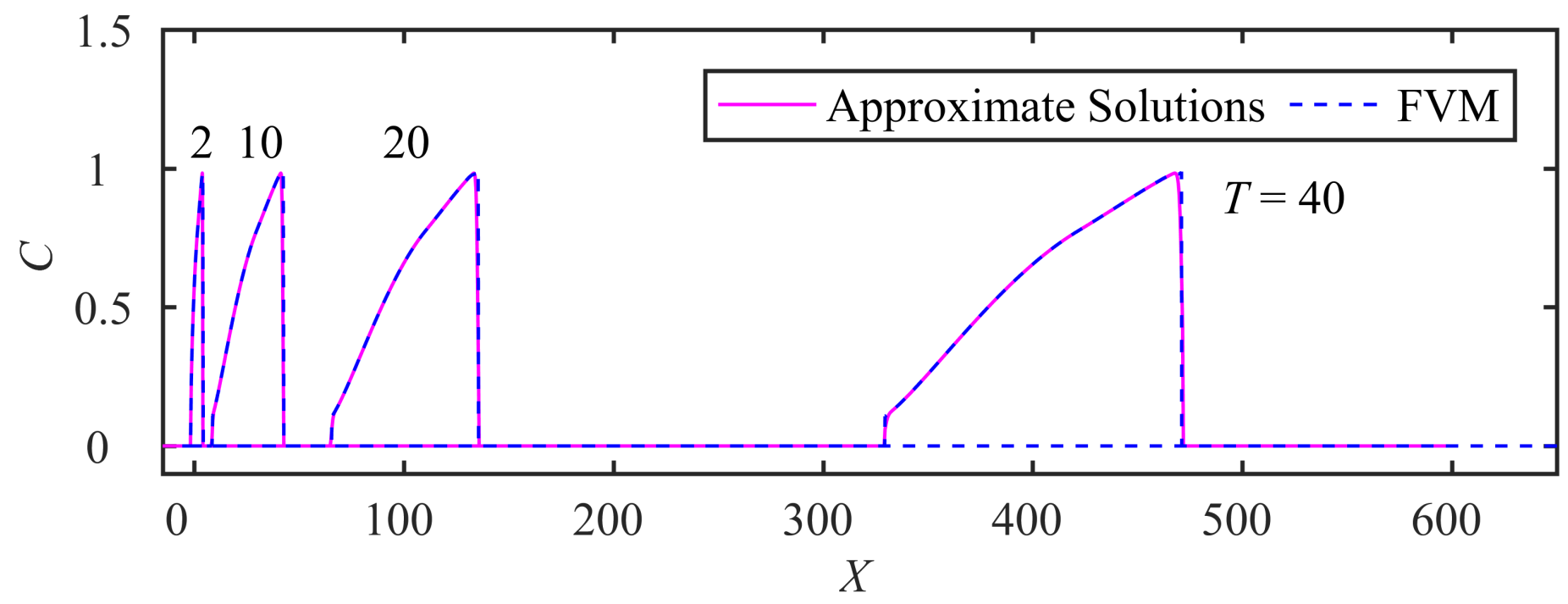


Figure 9. 


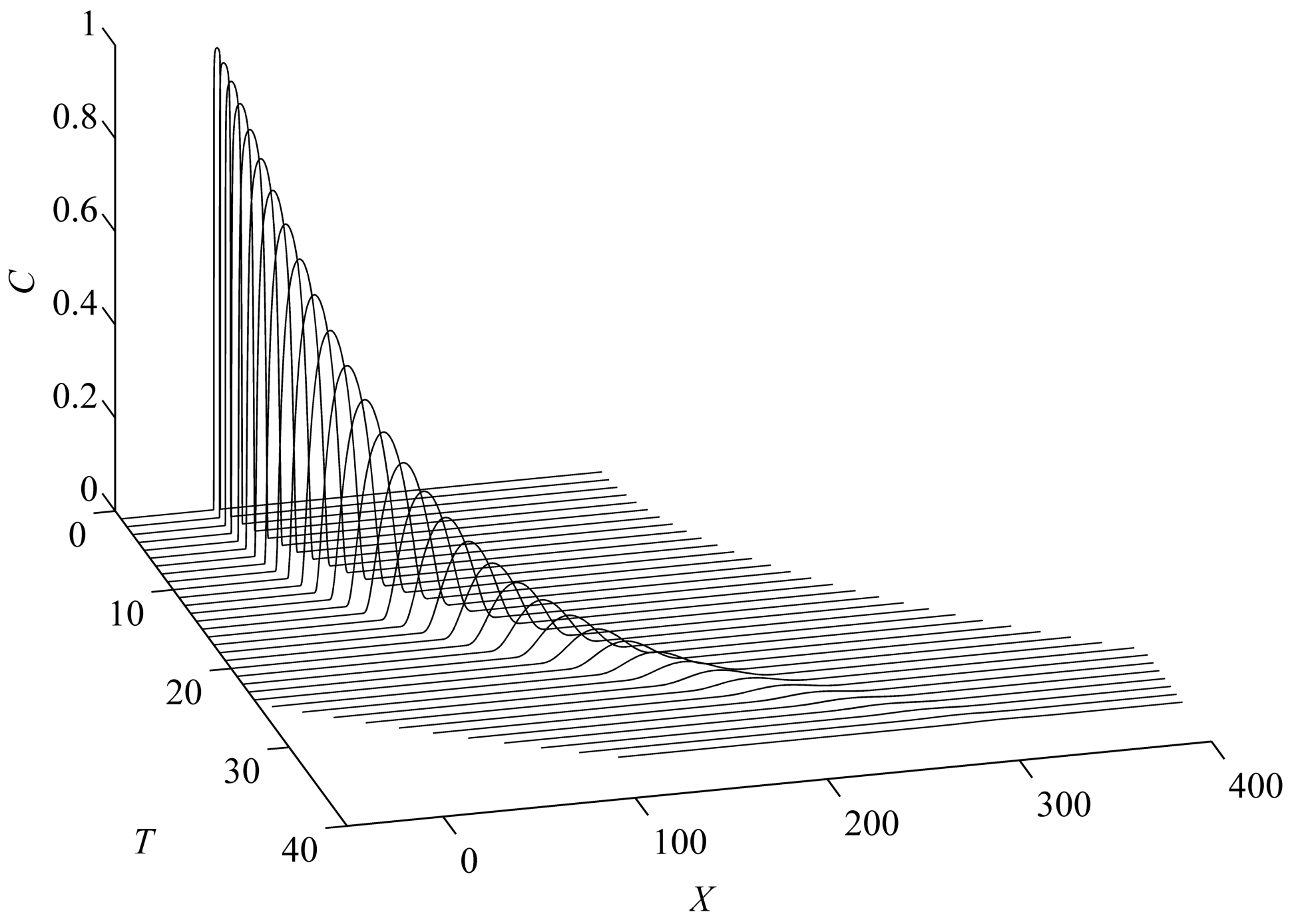


Figure 10. 
Approximate Solutions ---- FVM
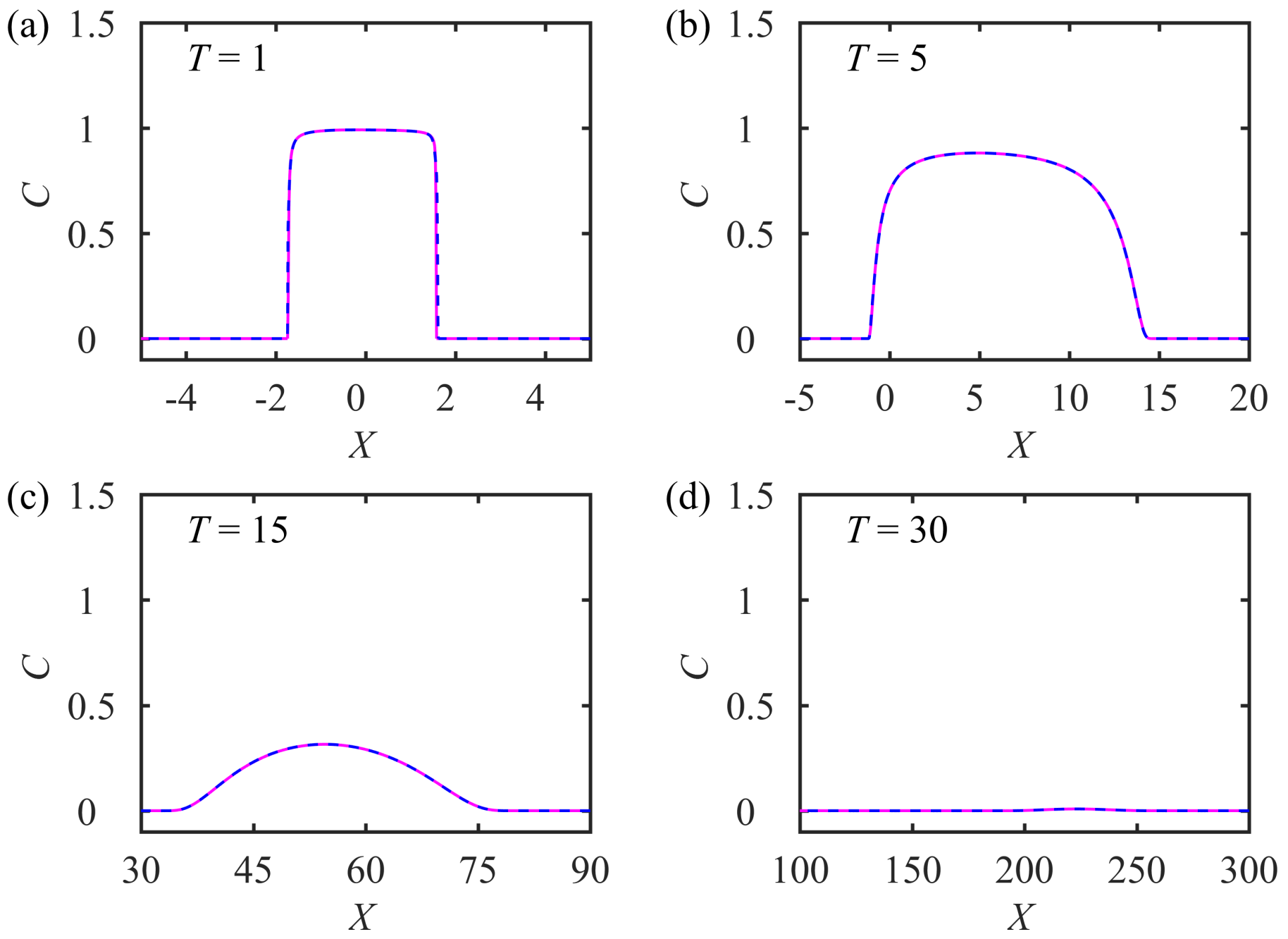
Figure 11. 


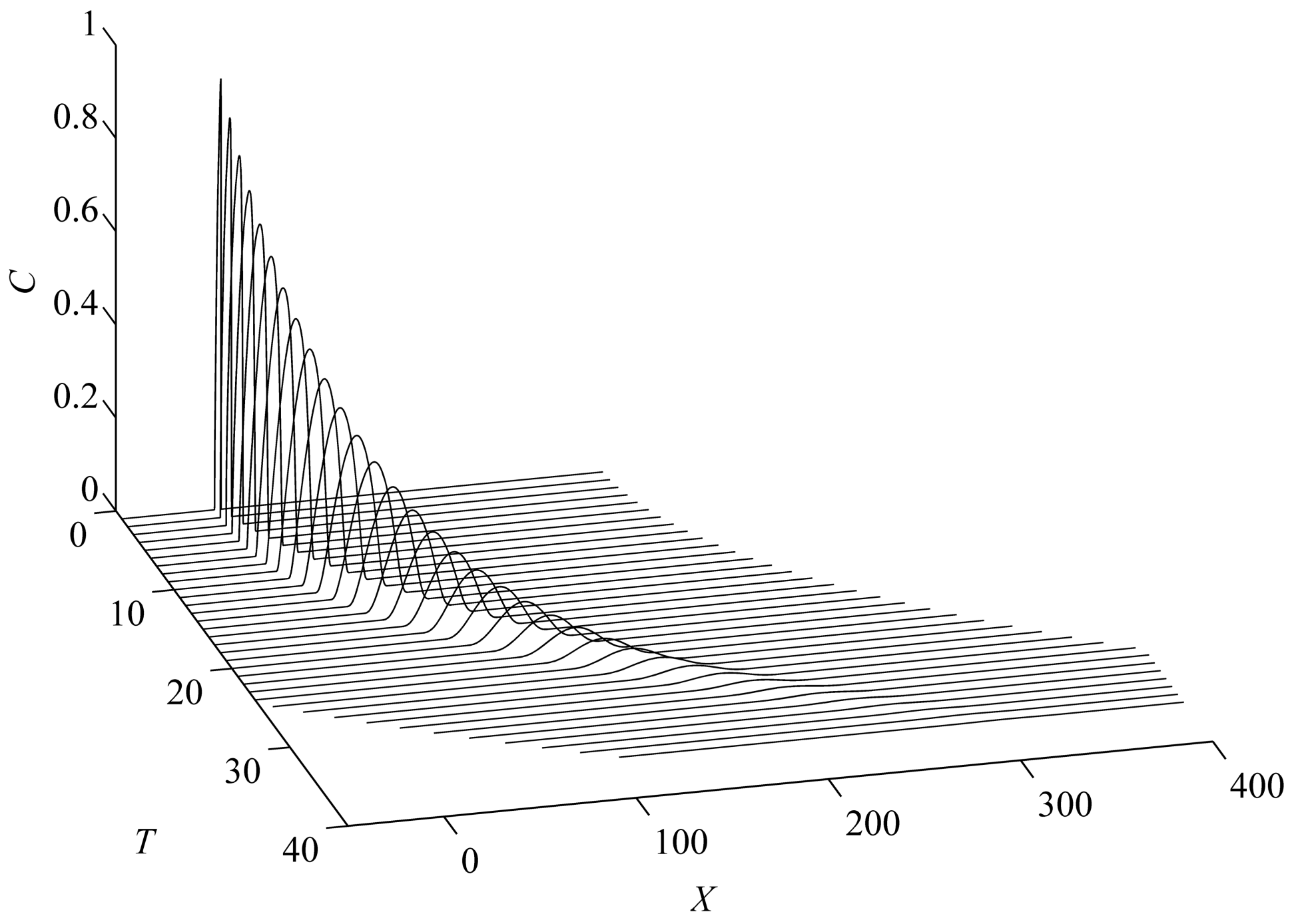


Figure 12. 


\section{Approximate Solutions ---- FVM}
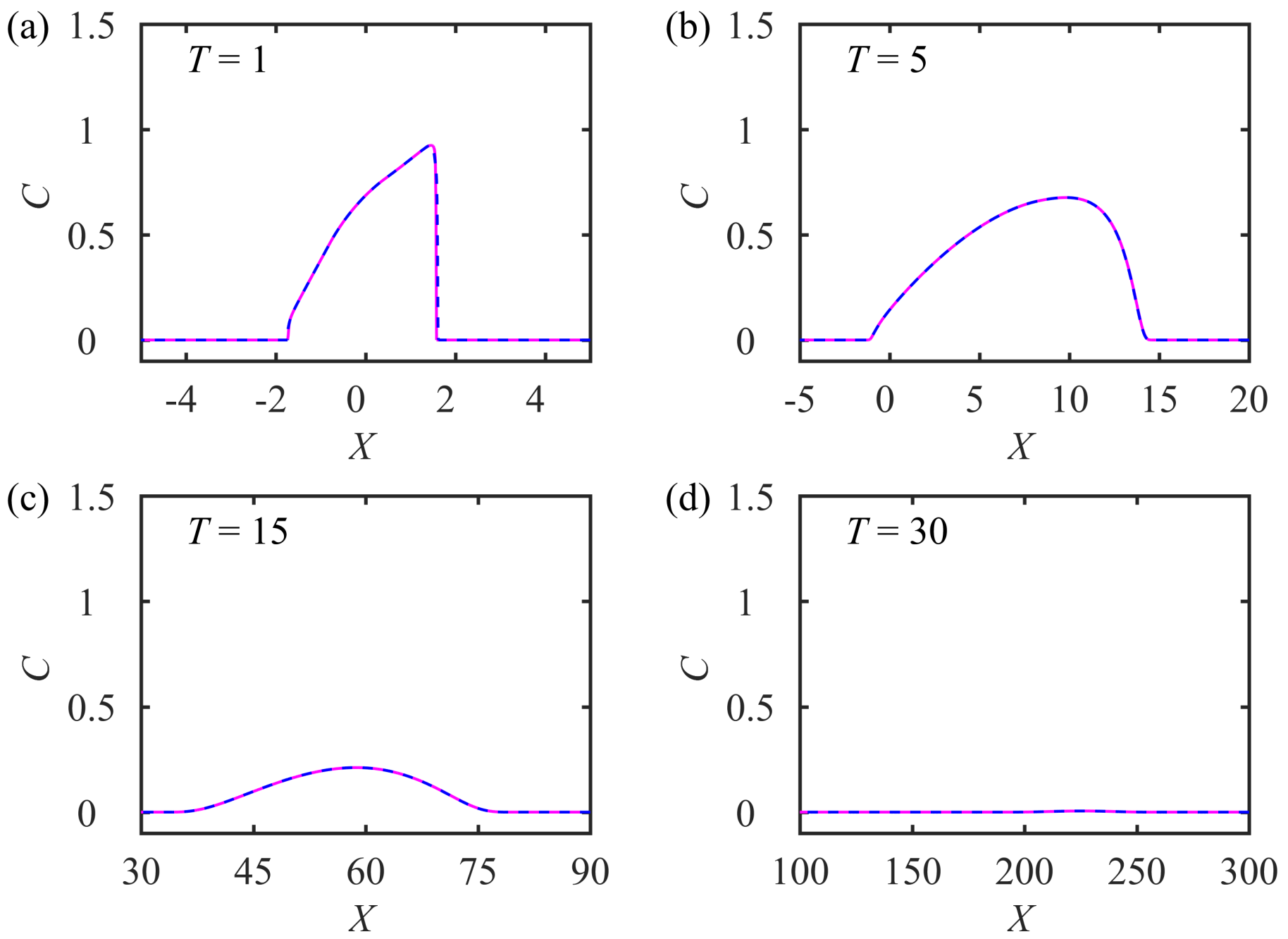
Figure 13. 


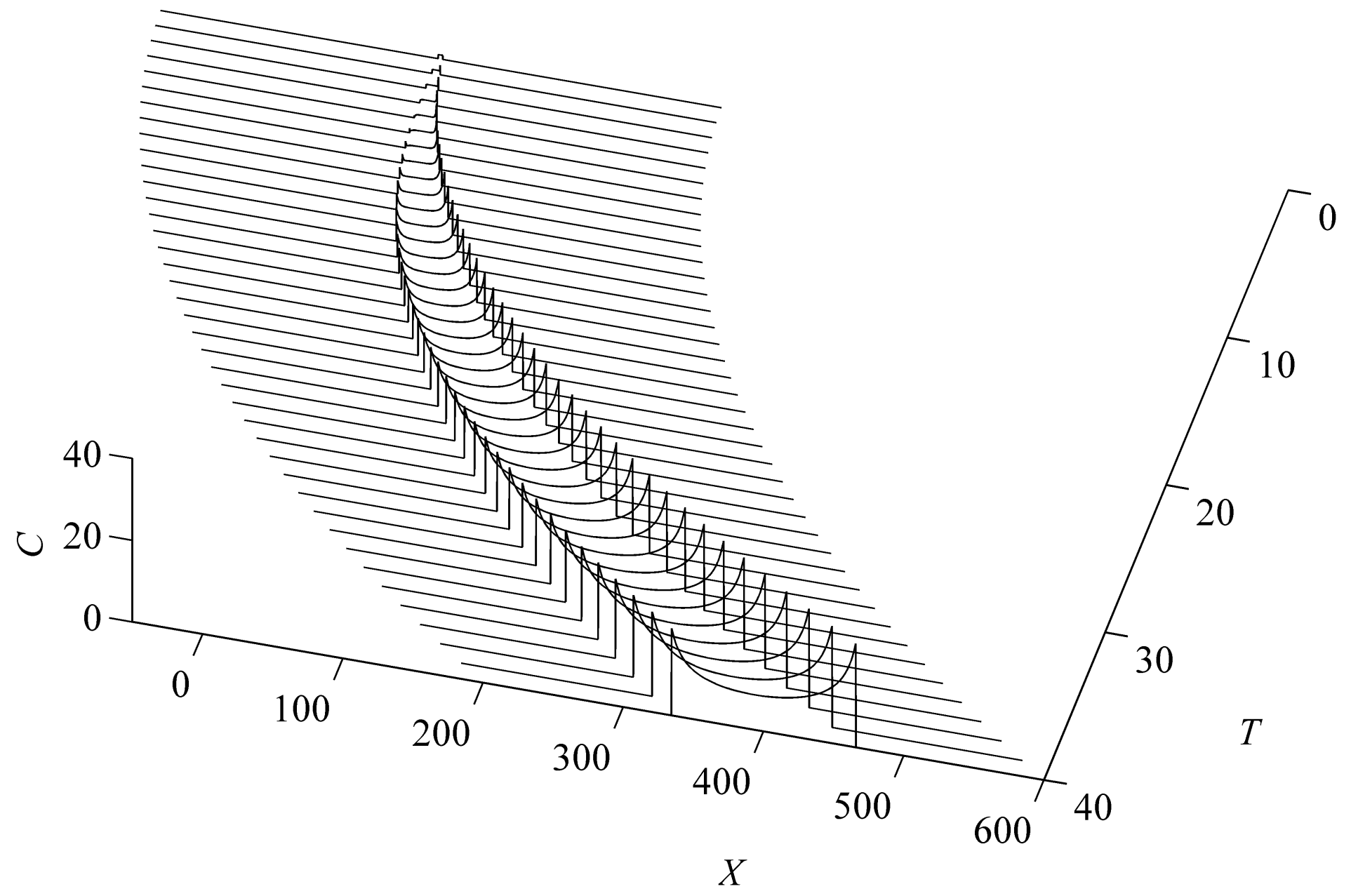


Figure 14. 


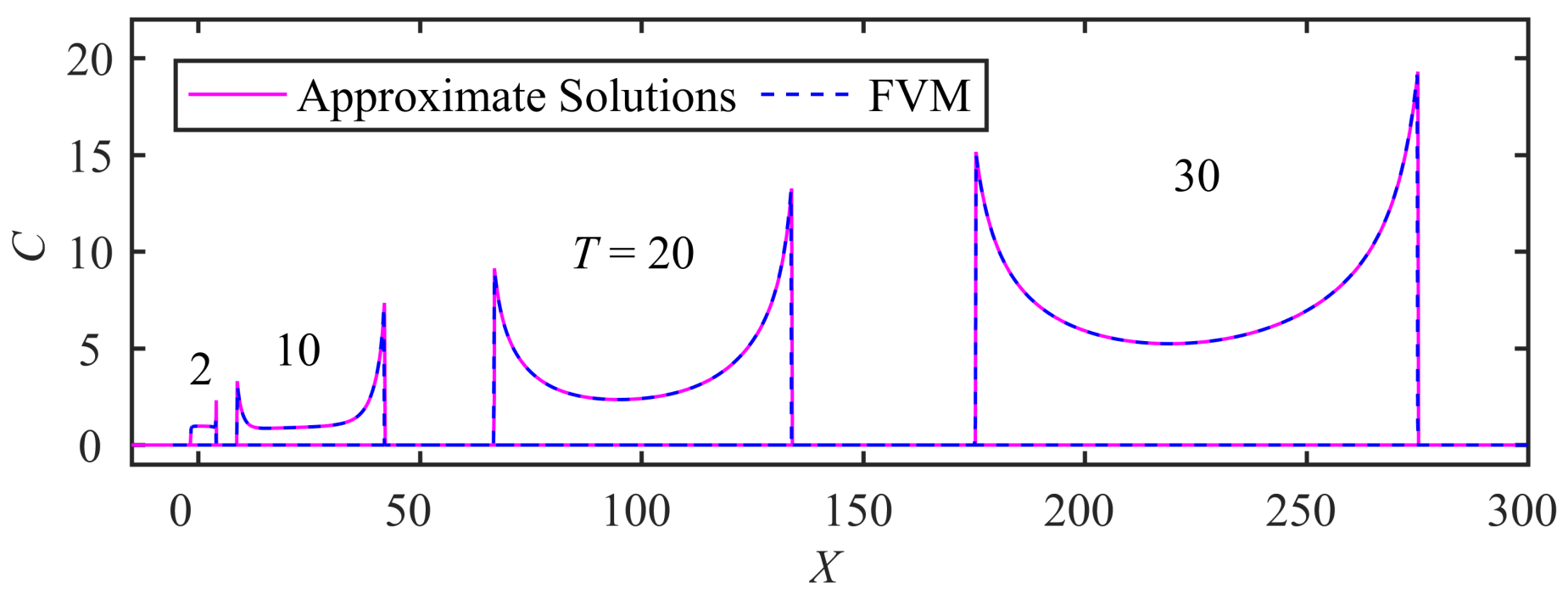


Figure 15. 


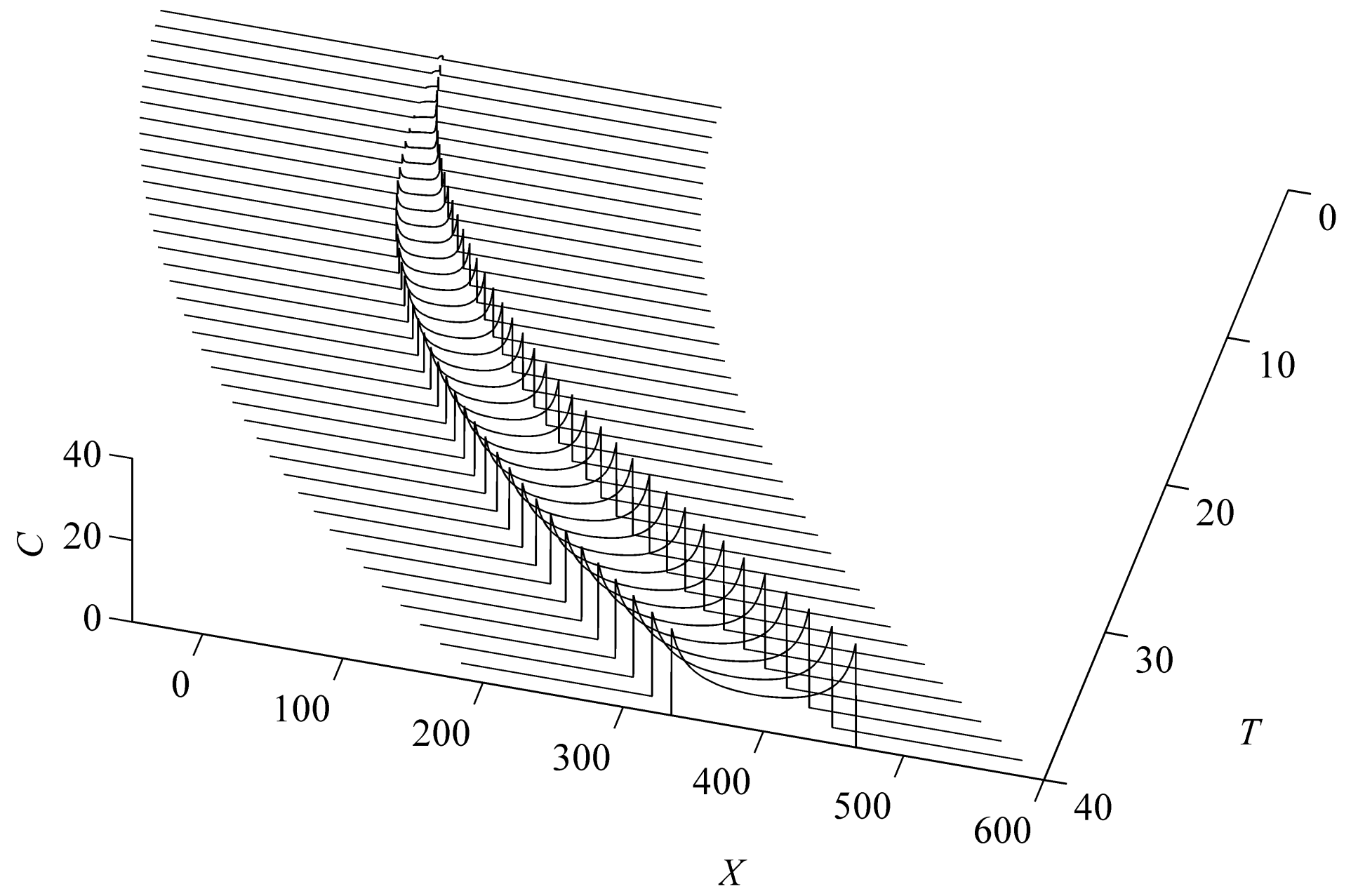


Figure 16. 


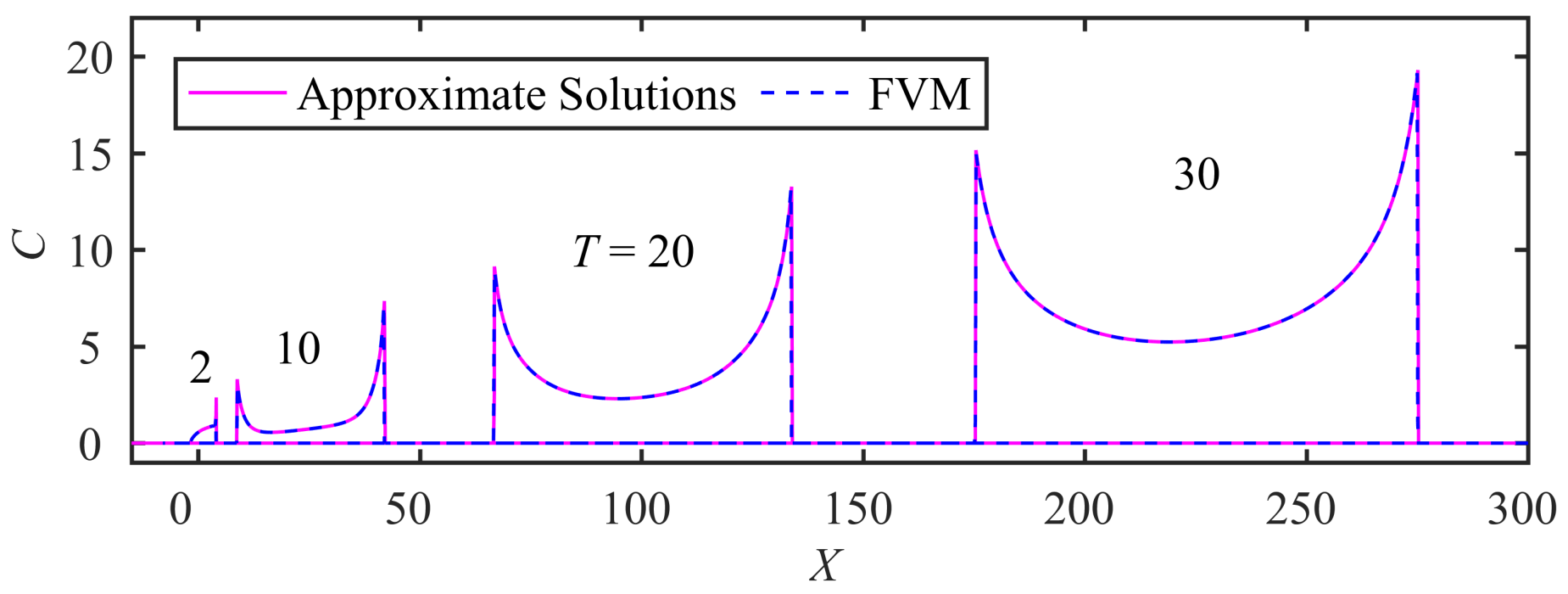


Figure 17. 


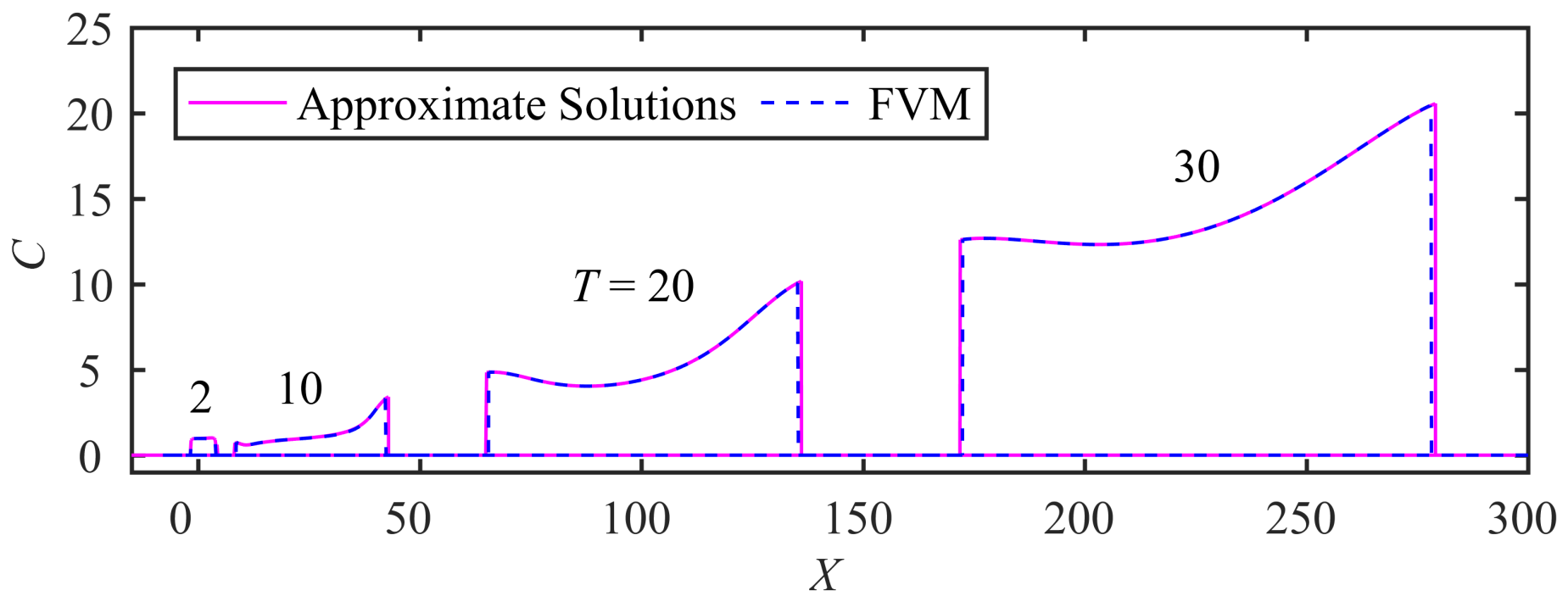


Figure 18. 


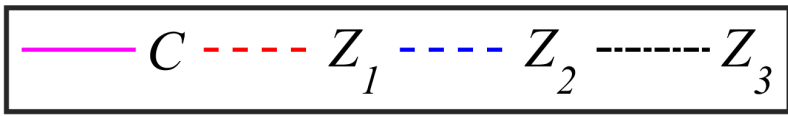
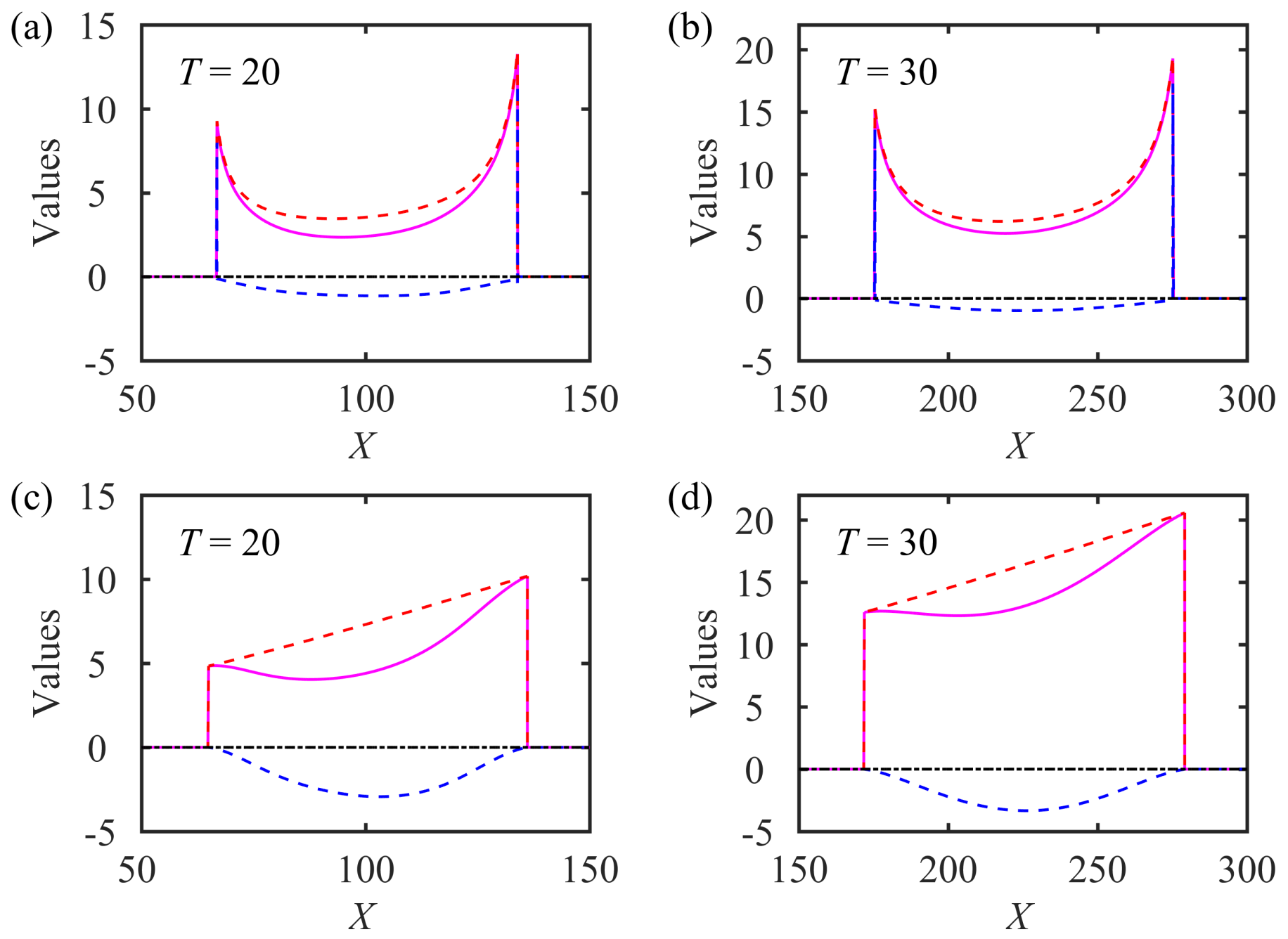